\title{
RNA-binding protein ZFP36L1 maintains posttranscriptional regulation of bile acid metabolism
}

\author{
Elizabeth J. Tarling, ${ }^{1,2,3}$ Bethan L. Clifford, ${ }^{1}$ Joan Cheng, ${ }^{1}$ Pauline Morand, ${ }^{1}$ Angela Cheng, ${ }^{1}$ Ellen Lester, ${ }^{1}$ Tamer Sallam, ${ }^{1}$ \\ Martin Turner, ${ }^{4}$ and Thomas Q. de Aguiar Vallim ${ }^{1,2,3,5}$ \\ 'Department of Medicine, Division of Cardiology, and ²Molecular Biology Institute (MBI), UCLA, Los Angeles, California, USA. ${ }^{3}$ UCLA Johnson Comprehensive Cancer Center (JCCC), Los Angeles, California, USA. \\ ${ }^{4}$ Laboratory of Lymphocyte Signalling and Development, The Babraham Institute, Babraham Research Campus, Cambridge, United Kingdom. ${ }^{5}$ Department of Biological Chemistry, UCLA, Los Angeles, California, USA.
}

\begin{abstract}
Bile acids function not only as detergents that facilitate lipid absorption but also as signaling molecules that activate the nuclear receptor farnesoid $X$ receptor (FXR). FXR agonists are currently being evaluated as therapeutic agents for a number of hepatic diseases due to their lipid-lowering and antiinflammatory properties. FXR is also essential for maintaining bile acid homeostasis and prevents the accumulation of bile acids. Elevated bile acids activate FXR, which in turn switches off bile acid synthesis by reducing the mRNA levels of bile acid synthesis genes, including cholesterol 7 $\alpha$-hydroxylase (Cyp7a1). Here, we show that FXR activation triggers a rapid posttranscriptional mechanism to degrade Cyp7a1 mRNA. We identified the RNA-binding protein Zfp36/1 as an FXR target gene and determined that gain and loss of function of ZFP36L1 reciprocally regulate Cyp7a1 mRNA and bile acid levels in vivo. Moreover, we found that mice lacking hepatic ZFP36L1 were protected from diet-induced obesity and steatosis. The reduced adiposity and antisteatotic effects observed in ZFP36L1-deficient mice were accompanied by impaired lipid absorption that was consistent with altered bile acid metabolism. Thus, the ZFP36L1dependent regulation of bile acid metabolism is an important metabolic contributor to obesity and hepatosteatosis.
\end{abstract}

\section{Introduction}

Primary bile acids are synthesized in the liver and stored in the gall bladder and, following a meal, are secreted into the intestine, where they facilitate lipid absorption $(1,2)$. Virtually all aspects of the enterohepatic circulation involving bile acid synthesis, secretion, conjugation, and resorption in the intestine are dependent on the nuclear receptor farnesoid X receptor (FXR) (encoded by Nr1h4), which is highly expressed in enterocytes and hepatocytes (1). Endogenous bile acids such as chenodeoxycholic acid (CDCA), deoxycholic acid (DCA), and cholic acid (CA) $(3,4)$ are known to activate FXR, while muricholic acid (MCA) is thought to function as an FXR antagonist (5). A number of FXR agonists, including bile acid analogs, are currently being tested in clinical trials as potential treatments for steatosis and cholestasis (6).

Bile acid levels are tightly regulated through a negative feedback loop and end-product feedback inhibition that was first described almost half a century ago (2). Such feedback is critical, as elevated levels of bile acids can be toxic and result in severe metabolic complications including cholestasis, diarrhea, lipid malabsorption, inflammation, and cancer (1). The mechanism for feedback inhibition of bile acid synthesis is highly complex but largely dependent on FXR. In response to elevated bile acid levels, FXR is activated, leading to signaling cascades in both the liver and intestine that result in decreased hepatic mRNA levels of cholesterol $7 \alpha$-hydroxylase (Cyp7a1), the rate-limiting enzyme of bile acid syn-

Conflict of interest: The authors have declared that no conflict of interest exists. Submitted: March 15, 2017; Accepted: July 26, 2017.

Reference information: J Clin Invest. 2017;127(10):3741-3754.

https://doi.org/10.1172/JCI94029. thesis (2), and Cyp8b1, which is required for the synthesis of CA (7). At present, the known mechanisms that lead to decreased Cyp7a1 expression following FXR activation are dependent on transcriptional repression. In one pathway, FXR activates the gene encoding the nuclear receptor SHP (NrOb2), a classic FXR target gene and transcriptional repressor (8). In a second pathway, FXR activation in intestinal enterocytes induces the expression and subsequent secretion of FGF15 (FGF19 in humans) into the circulation (9). FGF15/19 then binds to a heterodimeric receptor on hepatocytes to initiate a signaling cascade leading to transcriptional repression of Cyp7a1 (10). FGF15/19 signaling was also shown to require SHP (11). Defects in a number of genes in bile acid metabolism pathways are associated with diverse metabolic disturbances, consistent with the premise that maintaining proper bile acid homeostasis is critical for preventing metabolic diseases.

In the present study, we identify and characterize an FXRregulated posttranscriptional pathway that controls Cyp7a1 mRNA and bile acid levels. We identified ZFP36L1, also known as TIS11b (12), BRF1, or RNF162B, as a direct FXR target gene. ZFP36L1 is an RNA-binding protein (RBP) that has been shown to bind to adenylate-uridylate-rich (AU-rich) elements in the 3'-UTRs of specific cytokine mRNAs in immune cells to promote mRNA degradation (13). Here, we used synthetic and endogenous FXR agonists to demonstrate that activation of hepatic FXR leads to a rapid induction of hepatic Zfp36l1 mRNA and protein in vivo and in vitro. Our in vitro studies demonstrate that ZFP36L1 can target both human or mouse Cyp7a1 3'-UTRs. In addition, in vivo ZFP36L1 gain- and loss-of-function studies showed that ZFP36L1 regulates Cyp7a1 mRNA levels and alters bile acid levels. Finally, we demonstrate that mice lacking hepatic 

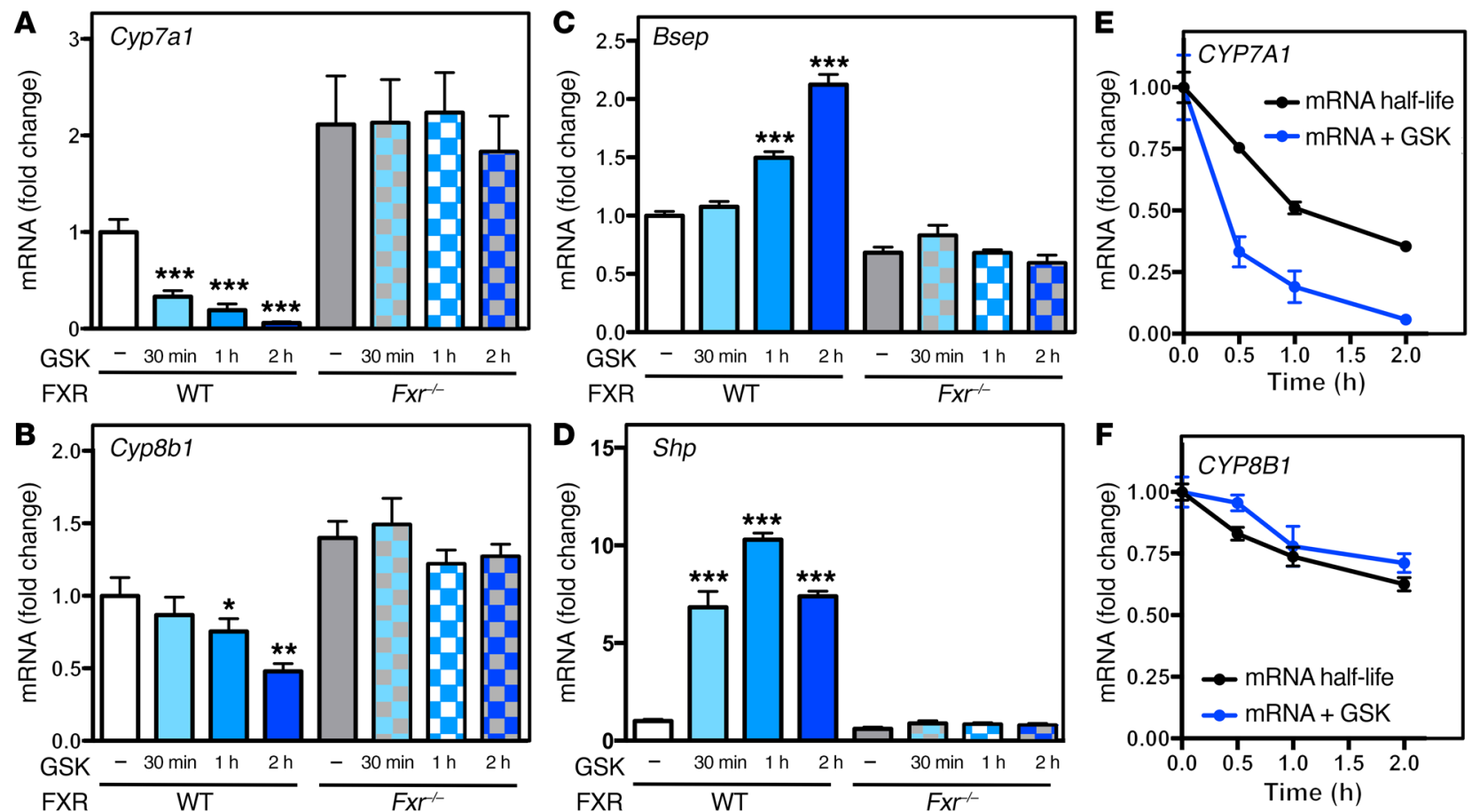

Figure 1. FXR activation leads to rapid changes in gene expression. (A-D) Hepatic gene expression of Cyp7a1, Cyp8b1, Shp, and Bsep in WT and Fxr ${ }^{-/-}$mice. Mice were treated with either vehicle $\left(n=16 \mathrm{WT} ; n=7 \mathrm{Fxr^{ \prime - } )}\right.$ or GSK2324, and RNA was isolated 30 minutes $\left(n=12 \mathrm{WT} ; n=6 \mathrm{Fxr^{ \prime - } )}, 1 \mathrm{hour}(n=10 \mathrm{WT} ; n=6\right.$ $\left.\mathrm{Fxr}^{-1}\right)$, or 2 hours ( $n=8 \mathrm{WT} ; n=7 \mathrm{Fxr}^{-1}$ ) after treatment, prior to sacrifice. Gene expression analysis was determined by qRT-PCR and normalized to Tbp. (E and F) Comparison of CYP7A1 and CYP8B1 mRNA levels in actinomycin D-treated IHHs (black lines) with Cyp7a1 and Cyp8b1 mRNA levels in mice treated with GSK2324 (blue lines indicate the same data as in A and B). Data represent the mean \pm SEM. ${ }^{*} P<0.05$, ${ }^{* *} P<0.01$, and ${ }^{* * *} P<0.001$, by 1 -way ANOVA.

Zfp36l1 (Zfp36l1-Ko) are partially resistant to diet-induced adiposity and steatosis, due, at least in part, to impaired lipid absorption that results from altered bile acid metabolism.

\section{Results}

Pharmacologic FXR activation with the synthetic FXR agonists GSK2324 or GW4064 results in a greater than 95\% reduction of Cyp7a1 and Cyp8b1 mRNA levels within 4 hours $(8,14)$. To better determine the kinetics of decay of Cyp7a1 and Cyp $8 b 1 \mathrm{mRNA}$ levels following FXR activation, we treated WT and $\mathrm{Fxr}^{\circ}$ - mice with a single dose of vehicle or GSK2324 (30 mg/kg body weight [mpk]) and analyzed gene expression 30 minutes, 1 hour, and 2 hours later. Cyp7a1 mRNA levels declined by more than $50 \%$ within 30 minutes of GSK2324 treatment of WT mice (Figure 1A). In contrast, Cyp8b1 mRNA levels decreased more gradually, reaching $50 \%$ expression levels after approximately 2 hours (Figure 1B). Neither mRNA decreased following GSK2324 treatment of $\mathrm{Fxr}^{\circ}$ - mice, in which basal expression was elevated compared with that in WT controls (Figure 1, A and B). As expected, 2 classic FXR target genes, Shp and Bsep, were induced in the livers of GSK2324-treated WT mice, but not in the livers of $\mathrm{Fxr}^{-}$mice (Figure 1, C and D), consistent with a requirement of $\mathrm{FXR}$ for the regulation of specific genes. The rapid decrease in the levels of Cyp7a1 and, to a lesser degree, of Cyp $8 b 1$ was specific to these key bile acid synthesis genes, since the remaining mRNAs encoding other enzymes of bile acid synthesis were not rapidly regulated following FXR activation (Supplemental Figure 1, A-K; supplemental material available online with this article; https://doi.org/10.1172/JCI94029DS1).
The rapid regulation of $C y p 7 a 1$ observed following FXR activation (Figure 1A) led us to consider the half-life of Cyp7a1 mRNA. A previous study in which HepG2 cells were used reported the halflife of human CYP7A1 to be 4.6 hours (15). Thus, our observation that Cyp7a1 mRNA levels decreased by approximately $50 \%$ within 30 minutes of GSK2324 treatment (Figure 1A) suggested that a posttranscriptional mechanism might enhance the degradation of preexisting Cyp7a1 mRNA following FXR activation in mice. To determine the half-life of Cyp7a1 mRNA in vivo is challenging, therefore we first considered using isolated primary mouse hepatocytes. However, we observed a near-complete loss of Fxr, Cyp7a1, and Cyp8b1 mRNAs within 16 hours of isolation and culturing of primary mouse hepatocytes (Supplemental Figure 1L), suggesting that these cells are not a suitable system by which to study the regulation of bile acid synthesis genes.

In the absence of primary hepatocytes or the appropriate murine cell lines that express Cyp7a1, we determined the halflife of CYP7A1 and CYP8B1 mRNA using immortalized human hepatocytes (IHHs) (16). IHHs express many of the bile acid synthesis genes, including CYP7A1 (16). We then treated IHHs with the transcriptional inhibitor actinomycin $\mathrm{D}$ for different lengths of time prior to isolation and quantification of mRNA levels to determine mRNA half-lives. The half-lives of human CYP7A1 and CYP8B1 mRNAs were approximately 1 and 4 hours, respectively (Supplemental Figure 1, M and N). We then compared the mRNA decay rates observed in IHHs (black lines) with those from GSK2324-treated mice (blue lines) and found that FXR activation with GSK2324 increased the rate of decrease in Cyp7a1, but not in 
A

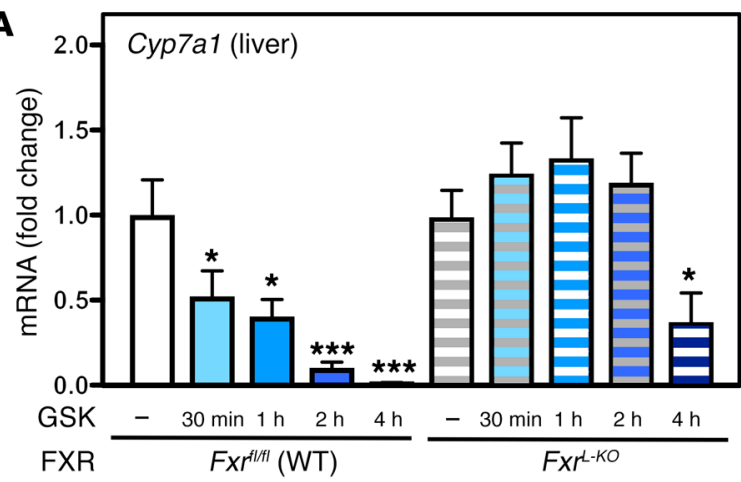

B

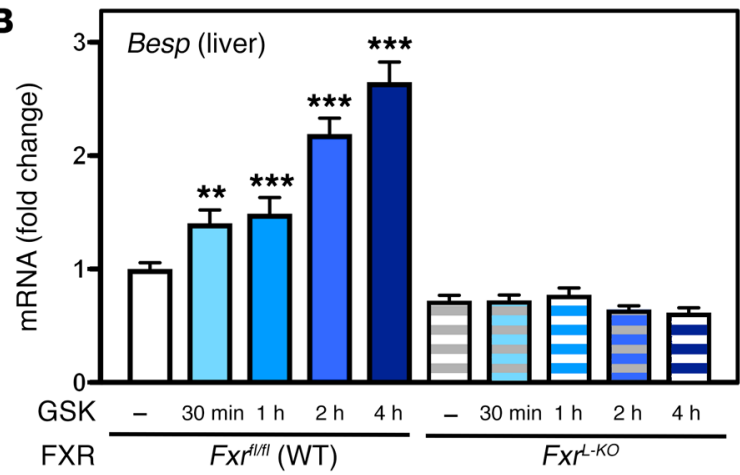

C

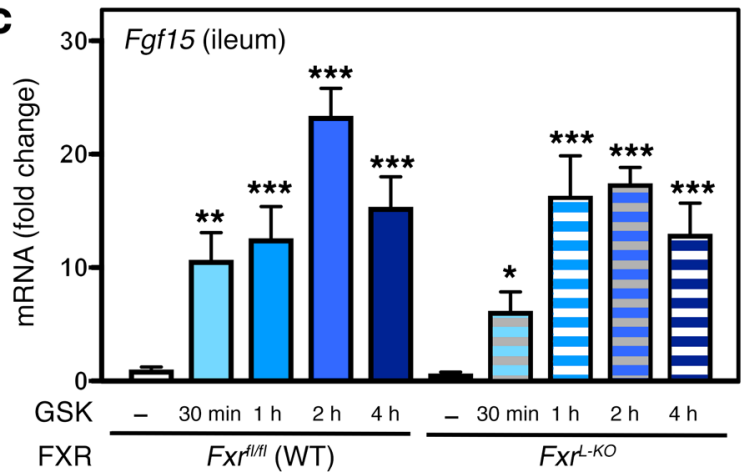

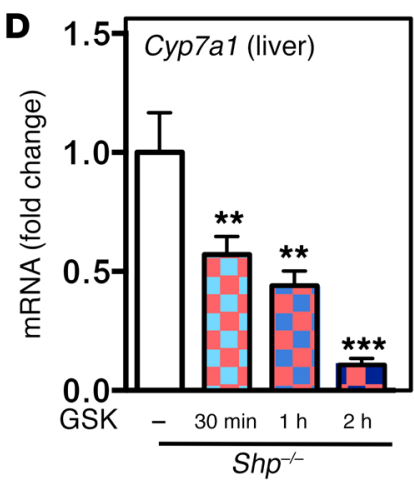

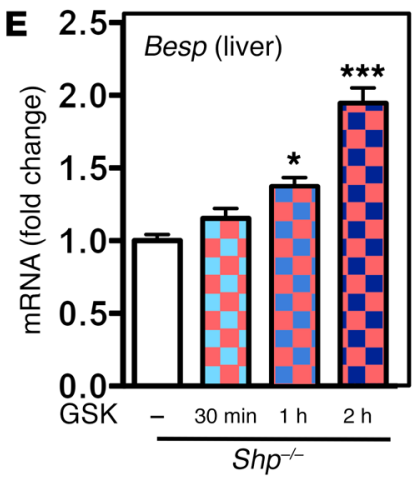

$\mathbf{F}$

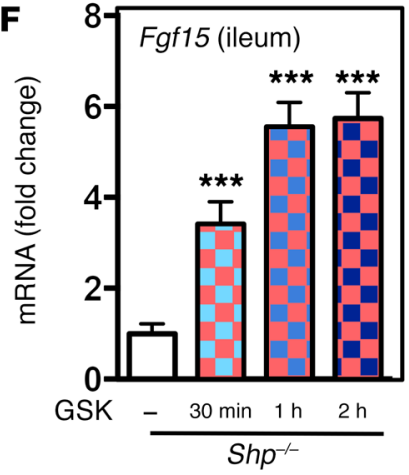

Figure 2. Rapid decrease in Cyp7a1 following FXR activation requires hepatic FXR but is independent of SHP. (A-C) Cene expression of hepatic Cyp7a1 and Bsep and ileal Fgf15 in littermate $F x r^{f l / f l}$ and $F x r^{1-k o}$ mice following treatment with either vehicle $\left(n=13 \mathrm{Fxr}^{f / / f f} ; n=11 \mathrm{Fxr^{-k }}{ }^{\mathrm{k} O}\right)$ or GSK2324 for 30 minutes $\left(n=9 F x r^{f / f f l} ; n=8 F x r^{L-K o}\right), 1$ hour $(n$

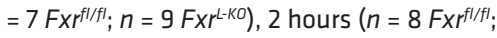
$\left.n=7 \mathrm{Fxr}^{L-K O}\right)$, or 4 hours $\left(n=5 \mathrm{Fx} \mathrm{r}^{\mathrm{fl} / \mathrm{fl} \text {; }}\right.$

$n=10 \mathrm{Fxr}^{\mathrm{L}-\mathrm{KO})}$. (D-F) Gene expression of hepatic Cyp7a1 and Bsep and ileal Fgf15 in $\mathrm{Shp}^{-/}$mice treated with either vehicle ( $n=$ 8 ) or GSK2324 for 30 minutes ( $n=8), 1$ hour $(n=7)$, or 2 hours $(n=8)$. Gene expression was determined by qRT-PCR and normalized to Tbp. Data represent the mean \pm SEM. ${ }^{*} P<0.05,{ }^{*} P<0.01$, and ${ }^{* *} P<$ 0.001 , by 1 -way ANOVA.
Cyp8b1, mRNA levels (Figure 1, E and F). The differences in mRNA half-lives between these conditions are suggestive of the existence of posttranscriptional mechanisms that destabilize Cyp7a1 mRNA.

Two pathways have been identified that are involved in feedback regulation of hepatic Cyp7a1 mRNA levels (1). One involves intestinally derived FGF15 (FGF19 in humans), an FXR target gene that is secreted into the circulation in response to activation of FXR in intestinal enterocytes (9). The second involves induction of SHP, a known transcriptional repressor, following activation of hepatic FXR (8).

To determine whether hepatic FXR is required for the rapid decrease in Cyp7a1 that occurs following FXR activation in vivo, we generated liver-specific FXR-KO mice $\left(F x r^{L-K O}\right)$ and littermate control floxed mice $\left(F x r^{f / f f}\right)$ by crossing $F x r^{f / / f l}$ mice with albumin-Cre-expressing mice to delete FXR in hepatocytes. We then treated both $F x r^{f / f f l}$ and $F x r^{L-K O}$ animals with either vehicle or GSK2324 for 30 minutes, 1 hour, 2 hours, and 4 hours. Cyp7a1 mRNA levels in the liver were reduced by $50 \%$ within 30 minutes, and the levels were almost completely abolished after 4 hours of GSK2324 treatment of Fxr $^{f / f l}$ mice (Figure 2A). In contrast, no inhibition of Cyp7a1 mRNA levels was observed for up to 2 hours after treatment of $F x r^{L-K O}$ mice with GSK2324 (Figure 2A). These data demonstrate that the initial and rapid GSK2324-dependent decrease in Cyp7a1 mRNA requires hepatic FXR. However, 4 hours after GSK2324 treatment of $\mathrm{Fxr}^{\mathrm{L}-\mathrm{KO}}$ mice, there was significant inhibition of Cyp7a1 mRNA (Figure 2A), suggesting that this latter inhibition is likely a result of intestinally derived FGF15.

Cyp8b1 mRNA levels were consistently reduced at a slower rate than were Cyp7a1 mRNA levels after GSK2324 treatment, and the initial decline in Cyp8b1 levels that occurred within 2 hours of GSK2324 treatment was dependent on hepatic FXR (Supplemental Figure 2A). The FXR target genes $S h p$ and Bsep were induced in the livers of $F x r^{f / f l}$, but not $F x r^{L-K O}$, mice treated with GSK2324 (Figure 2B and Supplemental Figure 2B). In contrast, GSK2324 treatment induced Fgf15 and Fabp6 (iBabp) mRNAs in the distal ileum in both Fxr $r^{f / f l}$ and $F x r^{L-K O}$ mice (Figure 2C and Supplemental Figure 2C), demonstrating that intestinal FXR and the subsequent induction of intestinal FXR target genes were unaffected by loss of hepatic FXR. These results suggest that a specific factor that is controlled by hepatic FXR mediates the rapid decline of Cyp7a1 mRNA levels following FXR activation. 


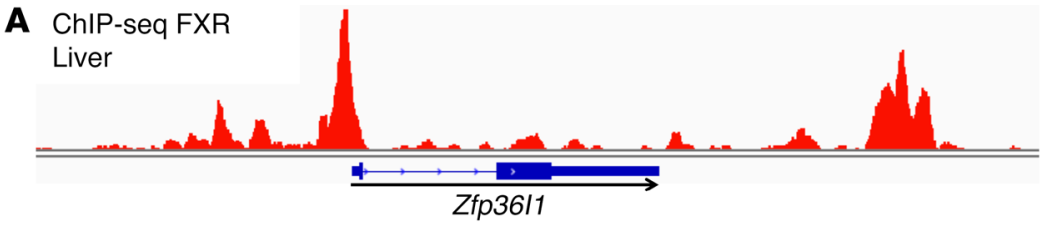

B

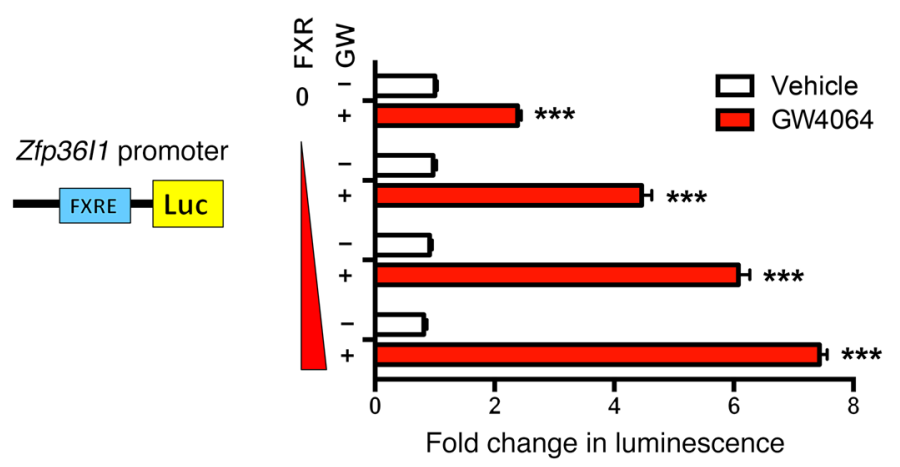

D

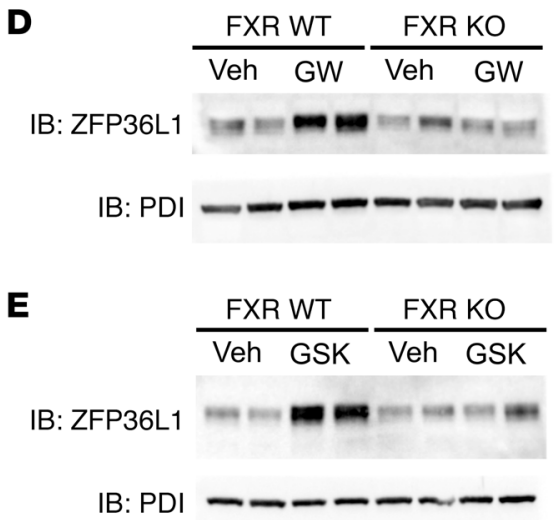

$\mathbf{F}$

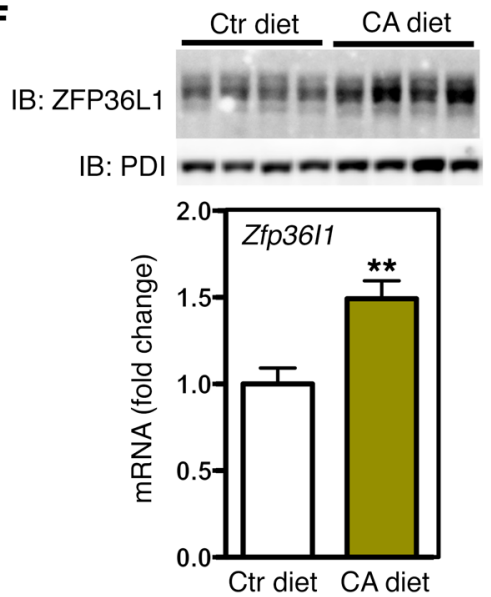

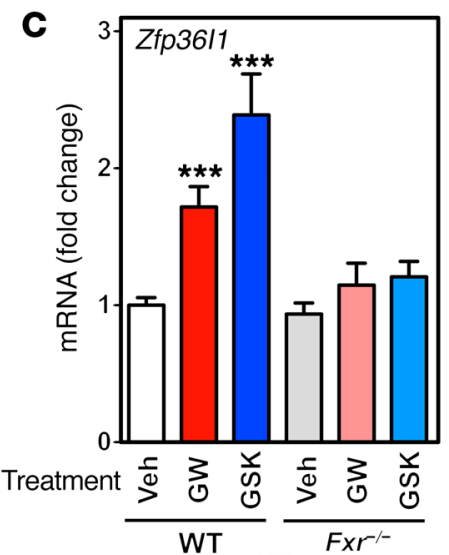

Figure 3. Zfp36/1 is a direct FXR target gene and is induced by both pharmacologic and physiologic FXR agonists. (A) FXR ChIP-seq analysis (21) of the Zfp36/1 genomic loci showing putative FXREs. (B) IHHs were transfected with a plasmid containing the Zfp36/1 promoter region ( $2 \mathrm{~kb}$ ) upstream of a luciferase reporter in combination with increasing amounts of a mouse Fxro2 expression plasmid. Cells were treated with vehicle or GW4064 (GW) $(1 \mu \mathrm{M})$ for 24 hours after transfection. Luciferase activity was normalized to $\beta$-gal and is expressed as the fold change ( $n=6$ wells/condition). ZFP36L1 mRNA (C) and protein (D and E) in C57BL/ 6 WT and $\mathrm{FXr}^{-1-}$ mice treated daily with vehicle, GW4064, or CSK2324 (60 mpk) for 3 days ( $n=7-9$ mice/group). (F) ZFP36L1 mRNA and protein in C57BL/6 WT mice fed either a control (Ctr) or $0.5 \%$ CA diet. Gene expression analysis was determined by qRT-PCR and normalized to Tbp, and protein analysis was determined by Western blotting, with PDI used as a loading control. Data represent the mean \pm SEM. ${ }^{*} P<0.01$ and ${ }^{* *} P<0.001$, by 1 -way ANOVA (B and $\mathbf{C})$ and Student's $t$ test (F). Veh, vehicle.
To determine whether the rapid decrease in Cyp7a1 mRNA observed in response to GSK2324 required the transcriptional repressor SHP, we obtained $\mathrm{Shp}^{-/}$mice (17) and treated them with vehicle or GSK2324 for 30 minutes, 1 hour, or 2 hours. Notably, Cyp7a1 mRNA levels declined by approximately $50 \%$ within 30 minutes of treating $\mathrm{Sh}^{-1 /}$ mice with GSK2324, indicating that SHP was not required for the rapid decrease in Cyp7a1 mRNA levels (Figure 2D). Treatment of $\mathrm{Sh}^{-/}$mice with GSK2324 also resulted in a decrease in Cyp8b1 mRNA after 2 hours (Supplemental Figure 2D). The induction of the known FXR target genes Bsep (Figure 2E) and Insig2a (Supplemental Figure 2E) in the liver and induction of Fgf15 in the distal Ileum (Figure 2F) were similar in $\mathrm{Sh}^{-/-}$mice, consistent with normal function of hepatic and intestinal FXR signaling in $\mathrm{Sh}^{-/-}$mice.

Taken together, these data demonstrate that the initial rapid decrease in Cyp7a1 mRNA levels in response to FXR activation in vivo requires hepatic FXR but is independent of both SHP and induction of intestinal Fgf15. Furthermore, our data suggested that activation of hepatic FXR utilizes a previously unrecognized posttranscriptional mechanism to control Cyp7a1 mRNA levels.

Various posttranscriptional mechanisms have been described that modulate mRNA stability: for example, the binding of micro-
RNAs (miRs) to seed sequences within target mRNA, or the interaction of the mRNA with specific RBPs $(18,19)$. We previously demonstrated that FXR regulates surprisingly few miRs. Among the direct FXR-regulated miRs we identified are the miR-144/miR-451 cluster (20) and miR-33 (21). Further, miRs are thought to be fine-tuners of gene expression and are therefore unlikely to be rapid regulators of gene expression (19). Thus, miRs seemed to be unlikely candidates for mediating the rapid change in Cyp7a1 mRNA. We next considered RBPs, which represent a large class of genes that are reported to be more abundant in the mammalian genome than are transcription factors (18). RBPs bind RNA targets and either stabilize or destabilize mRNAs, usually in a sequence-specific manner (18). Since FXR activation rapidly decreased Cyp7a1 mRNA levels, we hypothesized that FXR activation might result in the rapid induction of one or more specific RBPs that function to destabilize, and therefore enhance, the degradation of preexisting Cyp $7 a 1$ mRNA.

To test this hypothesis and to identify potential FXR-regulated RBPs, we searched the literature for RBPs that degrade mRNA targets and manually checked those for potential FXR response elements (FXREs) using publicly available FXR ChIP-sequencing (ChIP-seq) data sets $(22,23)$. This approach 

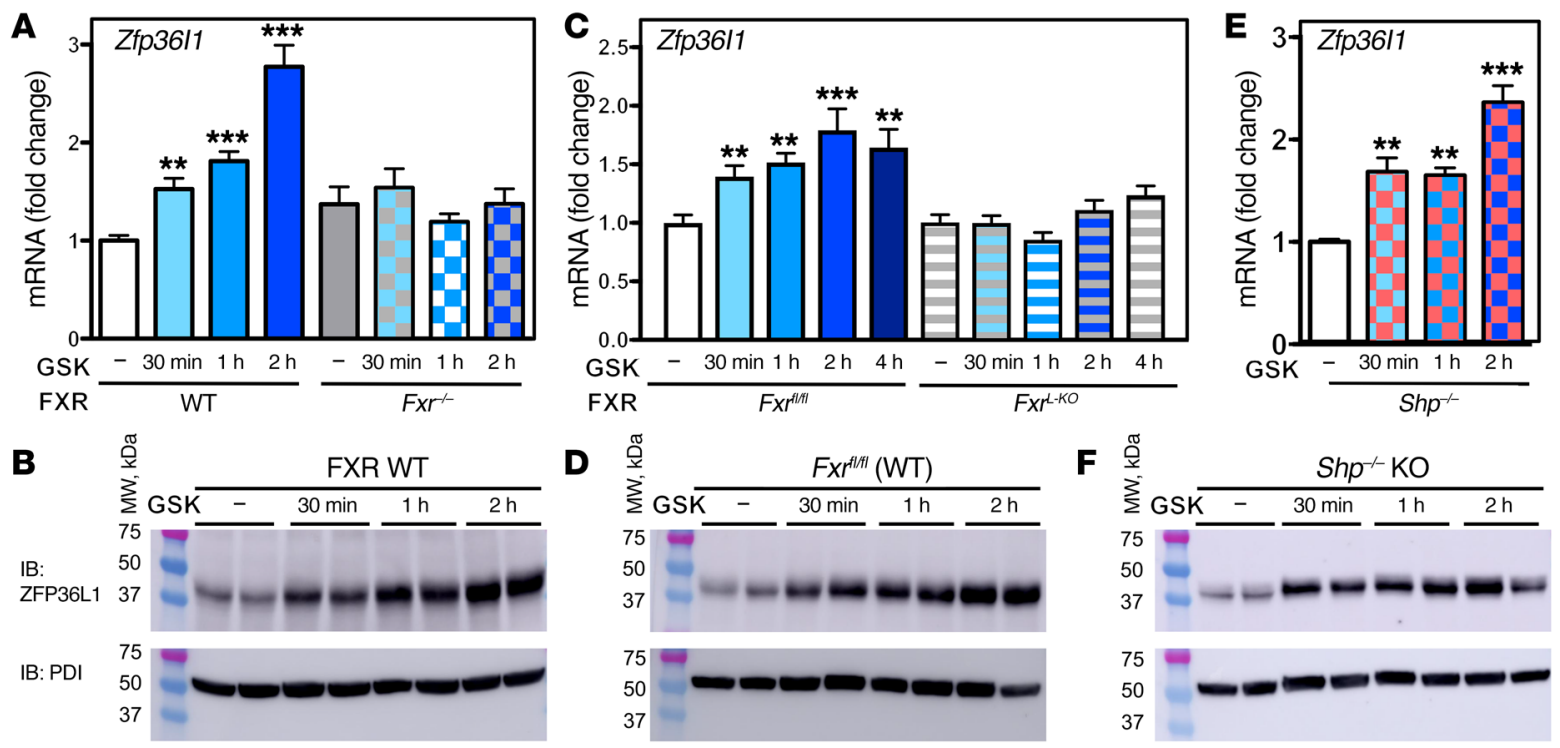

Figure 4. Zfp36/1 is rapidly induced following FXR activation with GSK2324. (A, C, and E) Zfp3611 mRNA and (B, D, and F) ZFP36L1 protein in the livers

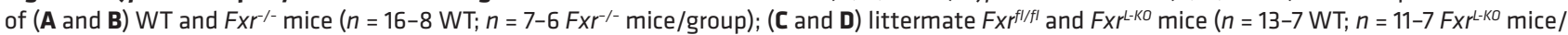
group); and (E and F) Shp ${ }^{-1-}$ mice ( $n=8-7$ mice/group) treated with either vehicle or GSK2324 for 30 minutes, 1 hour, or 2 hours. Gene expression analysis was determined by qRT-PCR and normalized to Tbp, and protein analysis was determined by Western blotting, with PDI used as a loading control. Data represent the mean \pm SEM. ${ }^{* *} P<0.01$ and ${ }^{* *} P<0.001$, by 1-way ANOVA. MW, molecular weight.

led us to investigate ZFP36L1, since liver FXR ChIP-seq analysis (22) identified several potential FXREs at the mouse Zfp36l1 locus. One FXRE is predicted to lie within the proximal promoter region of $Z f p 36 l 1$, while a second FXRE is predicted to lie downstream of the gene (Figure 3A). These data suggest that Zfp36l1 may be directly regulated by FXR.

To determine whether the putative FXRE in the Zfp36l1 promoter was functional, we cloned a $2-\mathrm{kb}$ region corresponding to the promoter of $Z f p 36 l 1$, upstream of a luciferase reporter gene. Cells were then cotransfected with this reporter gene in combination with increasing amounts of an FXR $\alpha 2$ expression plasmid and treated with either vehicle or the FXR agonist GW4064. The luciferase reporter driven by the mouse Zfp $36 l 1$ promoter was highly induced by FXR $\alpha 2$ and the presence of GW4064 in a dose-dependent manner (Figure 3B), suggesting that the promoter of Zfp36l1 contains functional FXREs.

To determine whether FXR activation results in the regulation of hepatic Zfp36ll in vivo, we treated WT and Fxr-- mice with either FXR agonist, GW4064 or GSK2324 (60 mpk), for 3 days. Zfp36l1 mRNA and protein were both induced in WT, but not $\mathrm{Fxr}^{-1}$, mice treated with either FXR agonist (Figure 3, C-E). To determine whether bile acids, which are endogenous FXR agonists, also regulate $Z f p 36 l 1$, we fed WT mice a diet supplemented with CA for 7 days and found that $C A$ feeding also led to an increase in hepatic Zfp36l1 mRNA and protein (Figure 3F, and see the complete unedited blots in the supplemental material). As expected, CA feeding resulted in increased Shp mRNA and robust reductions of Cyp7a1 and Cyp8b1 mRNA levels (Supplemental Figure 3, $A$ and $B$ ). To examine whether bile acids and other FXR agonists regulate ZFP36L1 mRNA in human cells, we treated IHHs with either endogenous (CDCA) or synthetic (GW4064) FXR agonists. Both agonists increased ZFP36L1 mRNA and protein levels and decreased CYP7A1 mRNA levels (Supplemental Figure 3, C and D). Taken together, these results show that FXR activation with either synthetic or endogenous agonists results in the induction of ZFP36L1 in both mouse livers and IHHs, consistent with ZFP36L1 as a direct FXR target gene.

We hypothesized that the regulation of ZFP36L1 by FXR at both mRNA and protein levels would have to be rapid if ZFP36L1 were to be involved in the initial (30 min) decrease in Cyp7a1 mRNA (Figures 1 and 2). To test this hypothesis, we determined how rapidly ZFP36L1 mRNA and/or protein levels were induced in WT, $\mathrm{Frr}^{-/}, \mathrm{Fxr}^{\mathrm{flff}}, \mathrm{Fxr}^{\mathrm{L}-\mathrm{KO}}$, and $\mathrm{Sh}^{-/-}$mice following short-term treatment with GSK2324. Importantly, we show that the induction of ZFP36L1 mRNA and protein was already observed 30 minutes after GSK2324 treatment of WT (Figure 4, A and B), Fxr ${ }^{f / / l}$ (Figure 4, C and D), and $S h p^{-/}$mice (Figure 4, E and F, and see the complete unedited blots in the supplemental material). In contrast, Zfp36l1 mRNA was not induced following GSK2324 treatment of $\mathrm{Fxr}^{-1}$ and $\mathrm{Fxr}{ }^{-\mathrm{KO} O}$ mice (Figure 4, A and C). We also determined the half-life of ZFP36L1 mRNA to be approximately 30 minutes in IHHs treated with actinomycin D, suggesting that $Z f p 36 l 1$ is a short-lived and rapidly regulated mRNA (Supplemental Figure $3 \mathrm{E})$. Finally, we show that ZFP36L1 expression was rapidly induced in IHHs treated with either GW4064 or CDCA (Supplemental Figure 3, F and G). Together, these in vitro and in vivo experiments demonstrate that activation of hepatic FXR results in a rapid (30 $\mathrm{min}$ ) increase in both ZFP36L1 mRNA and protein levels.

The stability of specific mRNAs has been shown to be dependent on the interaction of the $3^{\prime}$-UTR with various factors, including miRs and RBPs (18). Indeed, ZFP36L1 promotes mRNA degradation after binding to AU-rich sequences in the 3'-UTR of specific cytokines and then recruiting deadenylation complexes $(13,24)$. Interestingly, the Cyp7a1 3'-UTR is large (2.5 kb in mice, $1.5 \mathrm{~kb}$ in 
A

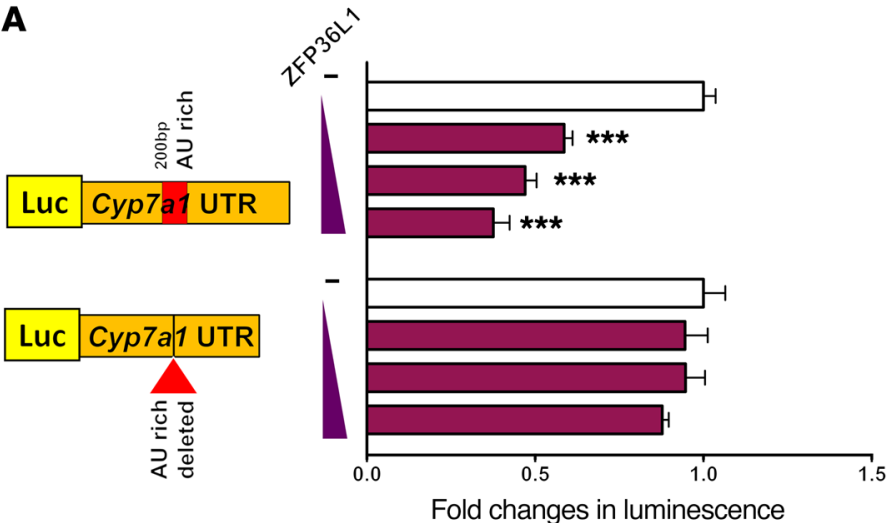

B

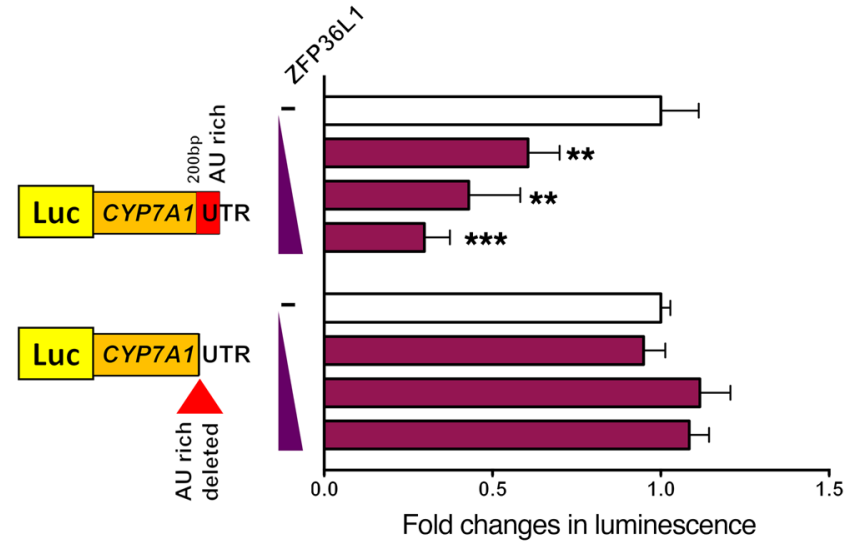

C

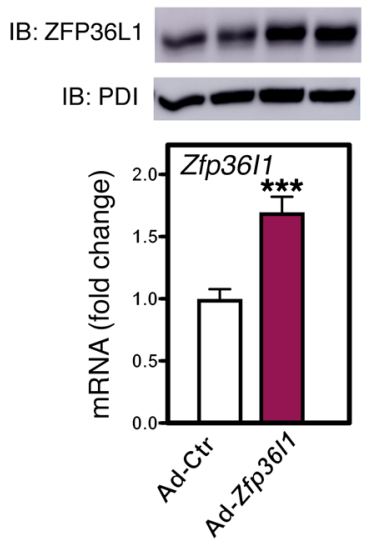

D

IB: CYP7A1

IB: PDI

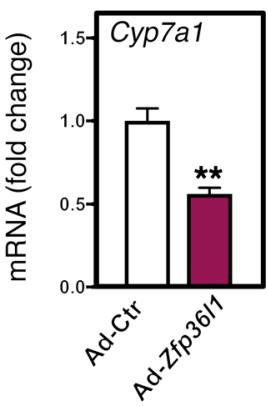

Figure 5. ZFP36L1 gain of function reduces CYP7A1 and bile acid levels in vivo. (A) Mouse and (B) human (WT and mutant) Cyp7a1 3'-UTR cloned downstream of a luciferase reporter and then transfected in IHHs in combination with increasing amounts of a mouse $Z f p 36 / 1$ expression plasmid. Luciferase activity was determined after 24 hours and normalized to $\beta$-gal and is expressed as the fold change ( $n=6$ wells/ condition). (C) ZFP36L1 mRNA and protein, (D) CYP7A1 mRNA and protein, and (E) mRNA levels of bile acid synthesis genes in male C57BL/6 WT mice treated with either adenovirus-control (Ad-Ctr) or Ad-Zfp36/1 ( $n=10$ mice/group). (F) Bile acid concentration in gall bladders and (C) plasma cholesterol levels in Ad-Ctr- or Ad-Zfp36/1-treated mice ( $n=10$ mice/group). Gene expression analysis was determined by qRT-PCR and normalized to Tbp. Data represent the mean \pm SEM. ${ }^{*} P<0.05$ ${ }^{* *} P<0.01$, and ${ }^{* *} P<0.001$, by 1-way ANOVA (A and $\mathbf{B}$ ) and Student's $t$ test ( C-C)
E

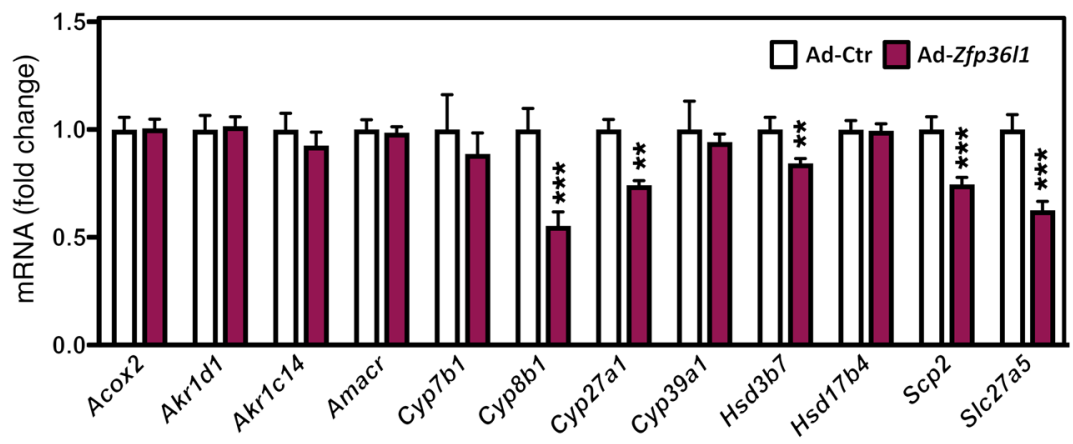

$\mathbf{F}$

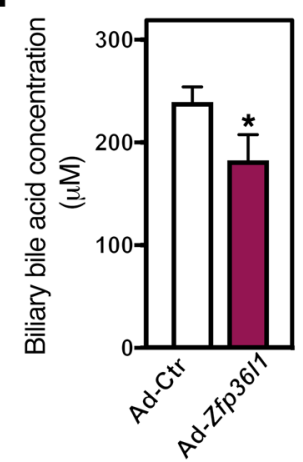

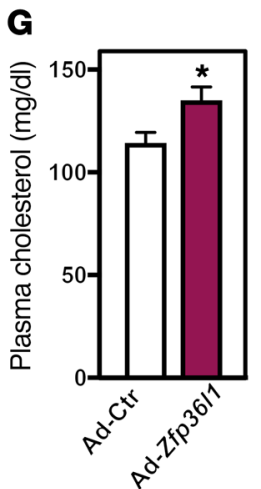

human) and contains multiple conserved AU-rich elements (24). To determine whether ZFP36L1 can regulate Cyp7a1 mRNA stability, we generated a reporter plasmid containing the mouse Cyp7a1 3'-UTR downstream of a constitutively transcribed luciferase reporter. Cotransfection of a plasmid encoding ZFP36L1, together with the Cyp7a13'-UTR reporter gene, resulted in a ZFP36L1 dosedependent decrease in luciferase activity (Figure 5A). The mouse Cyp7a1 3'-UTR has a 200-bp central region that contains several tandem AU-rich elements (Figure 5A). A reporter plasmid containing the mouse Cyp7a1 3'-UTR that lacked this 200-bp region was no longer responsive to coexpression of ZFP36L1 (Figure 5A). The human CYP7A1 3'-UTR also contains a similar 200-bp AU-rich sequence, which, in contrast to the mouse, is found at the $3^{\prime}$ end of the CYP7A13'-UTR. Cotransfection of ZFP36L1 with the luciferase reporter gene construct containing the human CYP7A13'-UTR also led to a decrease in luciferase activity (Figure 5B). Furthermore, deletion of the 200-bp AU-rich sequence from the 3'-terminus of the UTR resulted in a reporter gene that was unresponsive to cotransfected ZFP36L1 (Figure 5B). We conclude that ZFP36L1 targets both human and mouse CYP7A1 mRNA by binding to AU-rich elements in the $3^{\prime}$-UTR, leading to degradation of the mRNA.

To determine whether ZFP36L1 can also target endogenous Cyp7a1 mRNA in vivo, we generated Zfp36l1-expressing adenovirus particles and infused them into the tail veins of male C57BL/6 mice. Recombinant adenovirus particles resulted in a modest increase in hepatic ZFP36L1 mRNA and protein levels (Figure 5C). Consistent with our in vitro studies involving hybrid reporter genes, enforced ZFP36L1 expression in WT mice significantly 
A
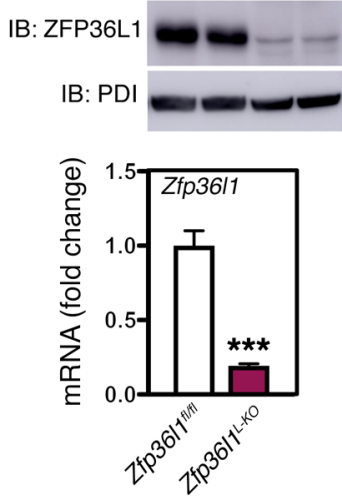

D

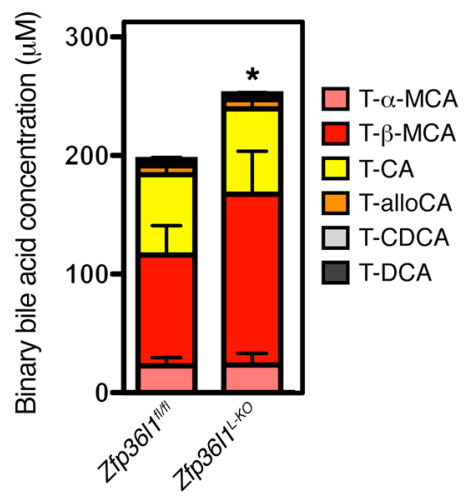

B

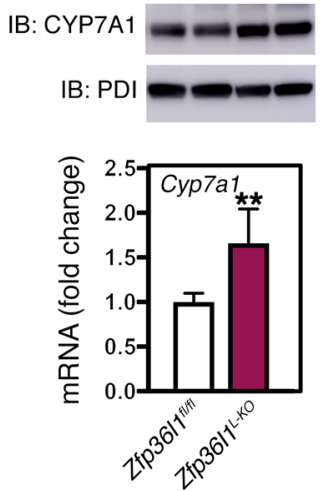

C

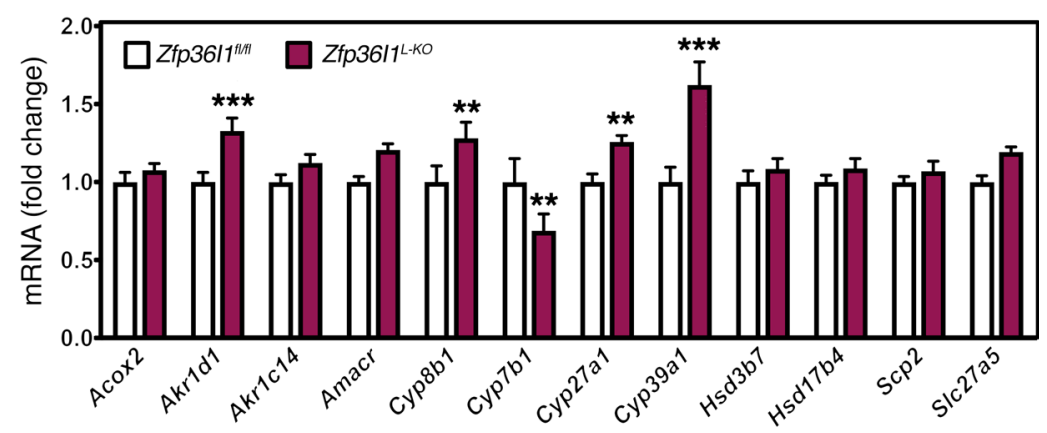

E

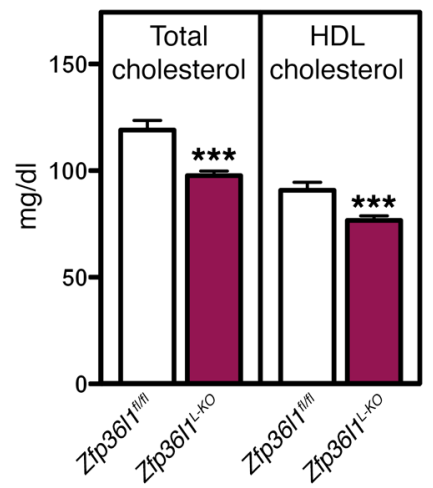

Figure 6. Zfp36I1-Ko mice have increased levels of CYP7A1 and bile acids in vivo. (A) ZFP36L1 mRNA and protein, (B) CYP7A1 mRNA and protein, and (C) mRNA levels of bile acid synthesis genes in male littermate Zfp36/7/flfl $(n=9)$ and Zfp36/1 $1^{L-K O}$ mice $(n=11)$. (D) Biliary bile acid concentration and bile acid composition and (E) plasma total and HDL cholesterol levels in littermate $Z f p 36 / 7^{f / f l}$ and $Z f p 36 / 1^{L-K o}$ mice. Gene expression analysis was determined by qRT-PCR and normalized to Tbp. Data represent the mean \pm SEM. ${ }^{*} P<0.05,{ }^{* *} P<0.01$, and ${ }^{* *} P<0.001$, by Student's $t$ test. decreased hepatic CYP7A1 mRNA and protein levels (Figure 5D, and see the complete unedited blots in the supplemental material).

We recently reported that expression of most of the 17 genes involved in the synthesis of bile acids from cholesterol is repressed following activation of FXR (14). We therefore examined whether these bile acid synthesis genes were also targeted by Zfp36l1. Enforced expression of ZFP36L1 in the livers of male mice led to the repression of some additional bile acid synthesis genes, namely Cyp8b1, Cyp27a1, Hsd3b7, Scp2, and Scl27a5 (Figure 5E). However, only Cyp7a1 and Cyp8b1 mRNA levels were decreased when female mice were treated with adenovirus expressing Zfp36ll (Supplemental Figure 4, A-C), suggesting that these 2 mRNAs were particularly sensitive to ZFP36L1.

CYP7A1 is the rate-limiting enzyme involved in the conversion of hepatic cholesterol to primary bile acids (2). Consequently, we next tested the hypothesis that the ZFP36L1-dependent decrease in CYP7A1 mRNA and protein resulted in decreased bile acids. Bile acids turn over very slowly, with only $5 \%$ of the bile acid pool being excreted per day, and this loss by excretion is replenished by de novo bile acid synthesis (1). As shown in Figure 5F, biliary bile acid concentration significantly decreased following 5 days of ZFP36L1 overexpression. The total amount of bile acids in the gall bladders of adenovirus-Zfp36l1-treated (Ad-Zfp36l1-treated) mice was also decreased, but the volume of bile recovered from the gall bladder was unchanged (Supplemental Figure 4, D and E).

We also determined other metabolic parameters in mice overexpressing $Z f p 36 l 1$. First, as shown in Figure 5G, plasma total cholesterol levels were significantly elevated following $Z f p 36 l 1$ overexpression. In contrast, plasma insulin and glucose levels were unaffected in mice following hepatic overexpression of Zfp36l1 (Supplemental Figure 4, F and G). Further, plasma levels of 2 liver enzymes, alanine aminotransferase (ALT) and aspartate aminotransferase (AST), were not altered following hepatic Zfp36l1 overexpression (Supplemental Figure 4, H and I). These results emphasize an important role of ZFP36L1 in the regulation of lipid metabolism, given that short-term gain of function of $Z f p 36 l 1$ in vivo results in reduced $C y p 7 a 1$ expression, decreased bile acids, and increased plasma cholesterol levels.

To complement our gain-of-function studies, we next investigated whether loss of $Z f p 36 l 1$ reciprocally affects bile acid metabolism. Germline deletion of $Z f p 36 l 1$ results in embryonic lethality $(25,26)$. Consequently, we obtained floxed $Z f p 36 l 1$ mice $\left(Z f p 36 l 1^{f / f l}\right)$ (27) and crossed them with albumin-Cre-expressing animals to generate hepatocyte-specific Zfp36l1-KO mice (Zfp36l1 ${ }^{L-K O}$ ). Zfp36l1 deletion in hepatocytes resulted in a greater than $80 \%$ reduction in total hepatic ZFP36L1 mRNA and protein levels (Figure 6A, and see the complete unedited blots in the supplemental material). The residual hepatic $Z f p 36 l 1$ mRNA detected in the $Z f p 36 l 1^{L-K O}$ mice is likely a result of $Z f p 36 l 1$ expression in nonparenchymal liver cells such as Kupffer, endothelial, and/or stellate cells.

We next investigated whether loss of ZFP36L1 in hepatocytes alters bile acid metabolism. Consistent with the hypothesis that ZFP36L1 normally functions to lower hepatic Cyp7a1 levels, CYP7A1 mRNA and protein levels were significantly elevated in the livers of $Z f p 36 l 1^{L-K O}$ as compared with levels in control $Z f p 36 l 1^{f l f l}$ mice (Figure 6B, and see the complete unedited blots in the supplemental material). mRNA levels of the majority of additional bile acid synthesis genes in the livers of $Z f p 36 l 1^{L-K O}$ 

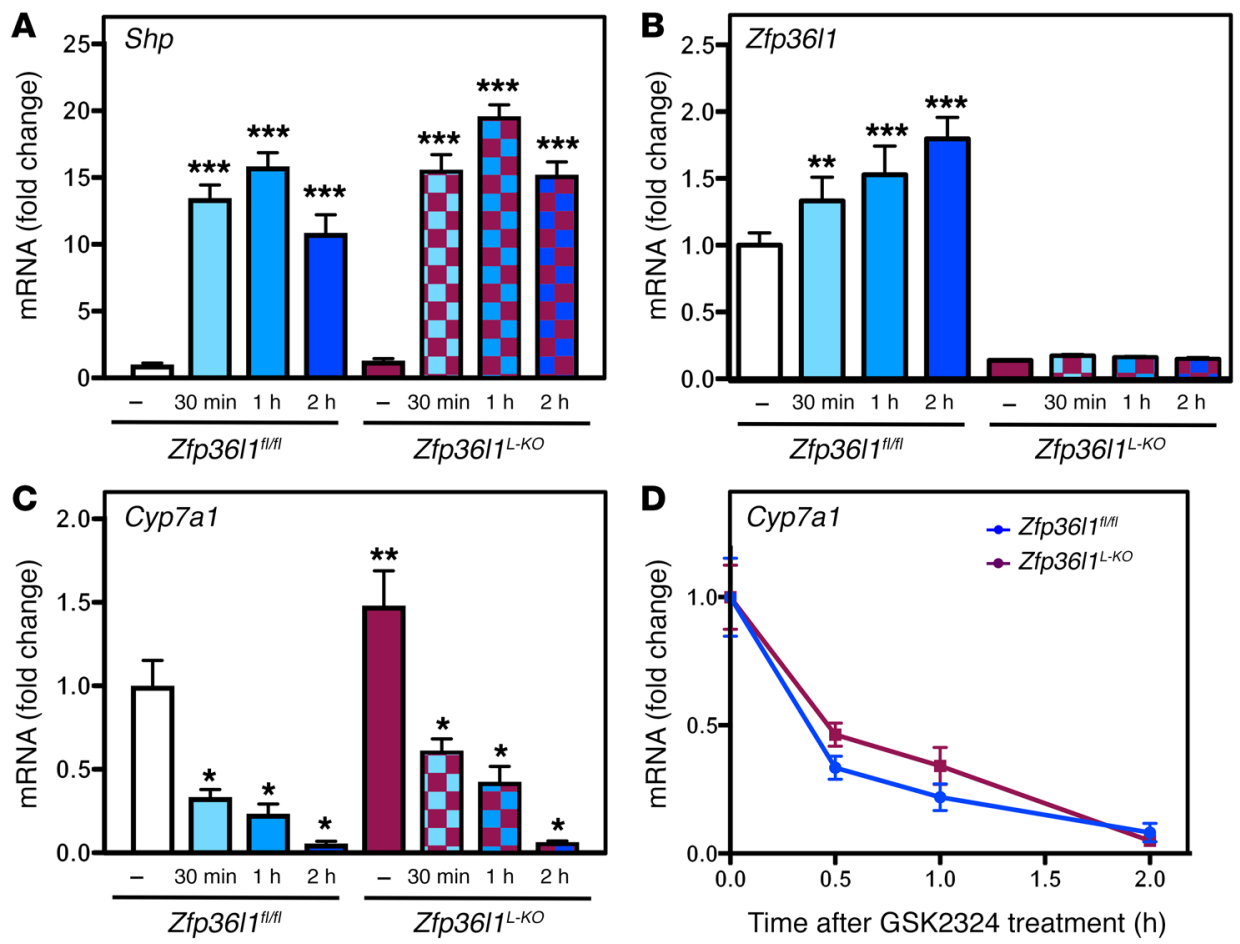

Figure 7. Redundant pathways controlled by FXR to regulate Cyp7a1. (A-C) Hepatic expression of Shp, Zfp36/1, and Cyp7a1 genes in $Z f p 36 / 7^{f / / f l}$ and littermate $Z f p 36 / 1^{-K O}$ mice treated with either vehicle ( $\left.n=10, Z f p 36 / 7^{f / f f} ; n=13, Z f p 36 / 1^{-K O}\right)$ or CSK2324 for 30 minutes $(n=8$, Zfp36/7 $\left.7^{f / f l} ; n=7, Z f p 36 / 1^{L-K o}\right), 1$ hour $\left(n=8, Z f p 36 / 1^{f / f \mid} ; n=8, Z f p 36 / 1^{L-k O}\right)$, or 2 hours ( $n=8, Z f p 36 / 7^{f / / f l} ; n=7$, Zfp36/1 ${ }^{-\mathrm{Ko}}$ ) before sacrifice. Gene expression analysis was determined by qRT-PCR and normalized to Tbp. (D) Cyp7a1 levels after GSK treatment in $\mathrm{Zfp} 36 / 7^{f / / f l}$ and Zfp36/1 ${ }^{-K O}$ mice compared with levels in vehicle-treated mice, set to 1 (blue lines represent the same data as in $\mathbf{C}$ ). (E) Summary diagram depicting known FXR-regulated pathways that control bile acid synthesis feedback inhibition. Data represent the mean \pm SEM. ${ }^{*} P<0.05$, ${ }^{* *} P<0.01$, and ${ }^{* *} P<0.001$, by 1-way ANOVA.

\section{E}

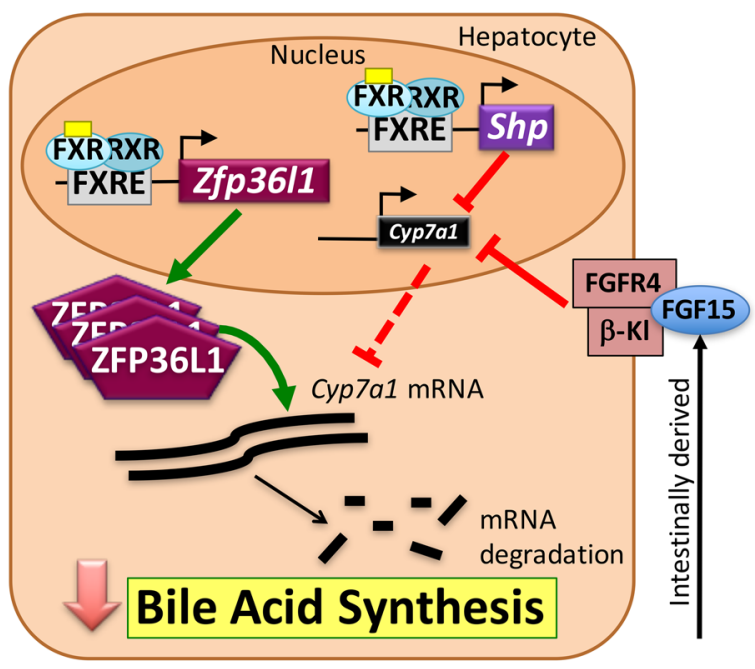

mice were unchanged, although some gene expression levels were increased (Akr1d1, Cyp8b1, Cyp27a1, and Cyp39a1), and Cyp7b1 mRNA expression was decreased (Figure 6C).

Total biliary bile acids were significantly elevated in Zfp36l1 ${ }^{L-K O}$ mice, and the increase was almost exclusive in 1 species of bile acid, namely tauro- $\beta$-muricholic acid (T- $\beta$-MCA) (Figure 6D). $Z f p 36 l 1^{L-K O}$ mice also had modestly larger gall bladders, resulting in a larger increase in the total amounts of biliary bile acid (Supplemental Figure 5, A and B). In line with increased bile acid production, $Z f p 36 l 1^{L-K O}$ mice also had decreased plasma levels of total and HDL cholesterol (Figure 6E). In contrast, the levels of plasma triglycerides (plasma TAG), free fatty acids, ALT, AST, insulin, and glucose were unaffected by loss of hepatic Zfp36l1 (Supplemental Figure 5, C-H). Finally, we detected no gross metabolic abnormalities in $Z f p 36 l 1^{L-K O}$ mice using indirect calorimetry. Oxygen consumption, carbon dioxide production, and activity were not significantly different in littermate $Z f p 36 l 1^{f l f l}$ and $Z f p 36 l 1^{L-K O}$ mice fed a standard rodent diet (Supplemental Figure 5, I-K).

Together, our combined in vivo gain- and loss-of-function studies strongly support an important role for hepatic ZFP36L1 specifically as a regulator of bile acid homeostasis. To determine whether the FXR/ZFP36L1 pathway is essential for the FXR-dependent regulation of bile acid synthesis, we carried out a time course of GSK2324 treatment of $Z f p 36 l 1^{f / f l}$ and littermate $Z f p 36 l 1^{L-K O}$ mice. Shp and Zfp36l1 mRNA levels were induced in $Z f p 36 l 1^{f / f l}$ mice, but only $S h p$ was induced in $Z f p 36 l 1^{L-K O}$ mice in response to the FXR agonist (Figure 7, A and B). Basal Cyp7a1 mRNA levels were elevated in vehicle-treated Zfp36l1-Ko mice (Figure 7B), consistent with the data shown in Figure 6. However, Cyp7a1 mRNA levels, which were rapidly decreased in $Z f p 36 l 1^{f / f l}$ mice treated with GSK2324, were still decreased in $Z f p 36 l 1^{L-K O}$ mice following GSK2324 treatment (Figure 7C). For direct comparison of the rates of Cyp7a1 mRNA degradation, we also show the data for both genotypes $\left(Z f p 36 l 1^{\Re / f l}\right.$ and $\left.Z f p 36 l 1^{L-K O}\right)$ as the fold change relative to the respective vehicle-treated animals (Figure 7D). These data show a modest delay in the loss of Cyp7a1 mRNA levels at 30 minutes and 1 hour following GSK2324 treatment of Zfp36l1 ${ }^{L-K O}$ as compared with $Z f p 36 l l^{f / f l}$ mice (Figure 7D). These latter findings suggest that additional FXR-dependent genes may encode other proteins that also function to degrade Cyp7a1 mRNA. Nonetheless, we conclude that the new pathway whereby FXR activation induces ZFP36L1 mRNA and protein, which subsequently targets Cyp7a1 mRNA for degradation, is not essential for feedback regulation of Cyp7a1. Our results suggest that multiple redundant and complementary pathways target Cyp7a1 via both transcriptional (SHP/FGF15) and posttranscriptional (ZFP36L1) mechanisms to ultimately block bile acid synthesis upon FXR activation (Figure 7E). 
Bile acids are both signaling molecules and detergents that facilitate lipid absorption, and mouse models with altered bile acid metabolism have a number of important metabolic phenotypes. For example, transgenic mice that overexpress human CYP7A1 in hepatocytes have increased bile acid synthesis but are also resistant to diet-induced obesity and steatosis (28-30). The precise mechanisms by which elevated CYP7A1 levels lead to changes in obesity or steatosis are not well understood. Given our observations that $Z f p 36 l 1^{L-K O}$ mice have elevated CYP7A1 mRNA and protein as well as increased bile acid levels (Figure 6), we hypothesized that loss of hepatic $Z f p 36 l 1$ would result in metabolic disturbances similar to those reported in CYP7A1-transgenic mice. We therefore challenged $Z f p 36 l 1^{L-K O}$ and littermate control $\left(Z f p 36 l l^{f l / f l}\right)$ mice with a Western-style diet ( $40 \mathrm{kCal}$ fat, $0.21 \%$ cholesterol) for 64 days and measured body weight and fat and lean mass weekly. $Z f p 36 l 1^{L-K O}$ animals fed the Western diet had less body weight gain and reduced fat mass (Figure $8, \mathrm{~A}$ and $\mathrm{B}$ ) as well as reduced lean mass (Supplemental Figure 6A) compared with littermate $Z f p 36 l 1^{f l / f l}$ mice. We obtained similar results when we fed $Z f p 36 l 1^{f / f l}$ and $Z f p 36 l 1^{L-K O}$ animals a diet only enriched in fat (high-fat diet: $45 \mathrm{kCal}$ fat, $0.03 \%$ cholesterol), and once again, $Z f p 36 l 1^{L-K O}$ mice were resistant to diet-induced weight gain (Supplemental Figure $6, \mathrm{~B}-\mathrm{D})$. To determine whether the reduced body weight and fat mass were due to differences in energy metabolism, we placed littermate $Z f p 36 l 1^{f l / f l}$ and $Z f p 36 l 1^{L-K O}$ animals in metabolic chambers and performed comprehensive metabolic analyses after 64 days of Western diet feeding. We observed no significant differences in energy expenditure (Figure 8C), oxygen consumption, carbon dioxide production, or activity (Supplemental Figure 6, E-G), suggesting that loss of hepatic Zfp36l1 did not result in gross energy metabolism abnormalities. However, despite exhibiting decreased body and fat weights, $Z f p 36 l 1^{L-K O}$ mice consumed more food than did their $Z f p 36 l 1^{f / f l}$ littermates (Figure 8D). Moreover, $Z f p 36 l 1^{L-K O}$ mice were markedly protected from diet-induced hepatosteatosis after 64 days on a Western diet (Figure 8E). Lipid extraction from the livers of control $Z f p 36 l 1^{f l / f l}$ and $Z f p 36 l 1^{L-K O}$ mice confirmed reduced hepatic triglyceride (TAG) and cholesterol levels in $Z f p 36 l 1^{L-K O}$ mice (Figure 8, F and G). Plasma TAG and cholesterol levels were also lower in the Western diet-fed $Z f p 36 l 1^{L-K O}$ mice as compared with levels in control mice (Figure 8, $\mathrm{H}$ and I), as were plasma insulin and ALT levels (Figure 8, J and K). Plasma glucose levels were lower, but not significantly, whereas plasma AST levels were not altered (Supplemental Figure 6, H and I). Hepatic expression of the inflammatory cytokines Il $1 b$ and Tnfa were also significantly lower in the livers of Western diet-fed $Z f p 36 l 1^{L-K O}$ mice (Supplemental Figure 6J). Together, these changes are consistent with improved hepatic function and improved metabolic handling in Zfp36l1 ${ }^{L-K O}$ mice following challenges with fat-enriched diets.

We also measured bile acid parameters in $Z f p 36 l 1^{L-K O}$ mice fed a Western diet. Total biliary bile acid levels were elevated in Western diet-fed $Z f p 36 l 1^{L-K O}$ mice, and the change was largely restricted to elevated T- $\beta$-MCA levels (Figure $8 \mathrm{~L}$ ). Moreover, Western diet-fed $Z f p 36 l 1^{L-K O}$ mice had increased hepatic CYP7A1 mRNA and protein levels and altered expression of a number of bile acid synthesis genes (Figure 8, M and N, and see the complete unedited blots in the supplemental material). These changes are consistent with our analysis of mice on a standard rodent diet (Figure 6).
Given the central role of bile acids as detergents, we hypothesized that loss of hepatic $Z f p 36 l 1$ might affect intestinal lipid absorption, particularly under Western diet-fed conditions, as a consequence of the altered bile acid metabolism. To determine differences in lipid absorption, we first subjected Western dietfed littermate $Z f p 36 l 1^{l / f l}$ and $Z f p 36 l 1^{L-K O}$ mice to an intragastric fat challenge and measured circulating triglycerides over a 6-hour period. $Z f p 36 l 1^{L-K O}$ mice had a reduced excursion of plasma TAG levels after the fat challenge, suggesting that loss of hepatic Zfp36l1 resulted in defective lipid absorption (Figure 80).

Reduced lipid absorption should also result in increased caloric content in the feces, and, indeed, the total energy in the form of calories that was recovered in the feces from $Z f p 36 l 1^{L-K O}$ mice was almost double that in feces from control mice (Figure 8P). Fecal calories were elevated even when expressed per gram, but the increased fecal calorie content was compounded by an increased fecal output by $Z f p 36 l 1^{L-K O}$ mice (Supplemental Figure 6, K and L). Taken together, these data suggest that loss of hepatic Zfp36l1 results in pronounced whole-body metabolic changes that are explained, at least in part, by decreased lipid absorption resulting from altered bile acid metabolism.

\section{Discussion}

Here, we identify $Z f p 36 l 1$ as an FXR target gene that functions to enhance the rate of degradation of Cyp7a1 mRNA. We used gainand loss-of-function studies to demonstrate that hepatic ZFP36L1 expression is inversely proportional to bile acid levels and, in addition, affects the composition of the bile acid pool. Thus, the current findings identify and characterize a previously unrecognized pathway by which activated FXR rapidly regulates bile acid synthesis. We show that FXR activation in vivo results in an intensely rapid decrease in Cyp7a1 mRNA by a posttranscriptional mechanism that requires hepatic FXR but is independent of the transcriptional repressor SHP. To determine the molecular mechanism of the degradation of Cyp7a1 mRNA, we identified the RBP Zfp36l1 (12). The Zfp36l1 gene locus contains multiple putative FXR response elements, consistent with its induction in response to natural (CA) or synthetic (GW4064, GSK2324) FXR agonists in vivo. Further, reporter gene assays indicated the presence of a functional FXRE in the proximal promoter of the $Z f p 36 l 1$ gene. Importantly, we show that in mice, induction of $Z f p 36 l 1 \mathrm{mRNA}$ and protein in response to FXR activation with GSK2324 occurs within 30 minutes. Taken together, these data suggest that rapid FXR-dependent induction of $Z f p 36 l 1$ leads to a reduction of $C y p 7 a 1 \mathrm{mRNA}$ via a posttranscriptional mechanism, a hypothesis supported by our in vitro reporter gene studies.

We show that luciferase reporter genes linked to either the mouse or human Cyp7a1 3'-UTRs are repressed following overexpression of ZFP36L1. Additional reporter gene assays indicate that ZFP36L1-dependent regulation requires a region containing conserved AU-rich elements in the 3'-UTR of Cyp7a1 mRNA. Indeed, previous studies have shown that $Z f p 36 l 1$ functions to repress cytokine and cell-cycle mRNAs by binding to AU-rich sequences in their 3'-UTRs $(13,27,31)$. However, a role for Zfp36l1 in regulating hepatic mRNAs in vivo, including those involved in lipid and bile acid metabolism, has not been reported. Notably, cytokine mRNAs were unaffected in the liver following treatment of mice with Ad-Zfp36l1 or in Zfp36l1 ${ }^{L-K O}$ mice (data not shown). 
A

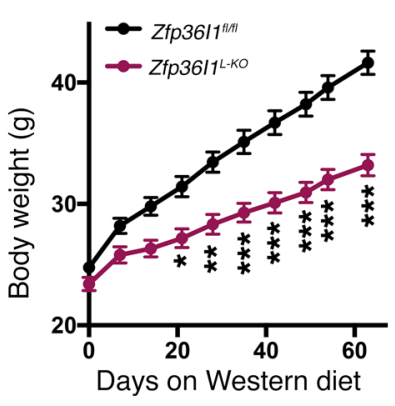

E

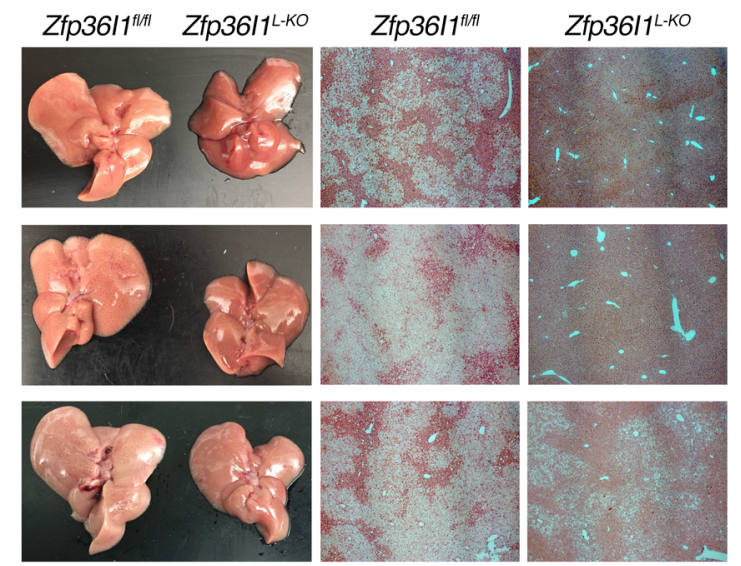

C

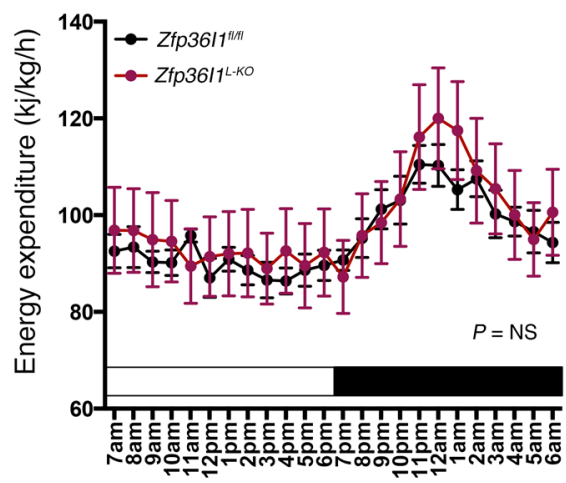

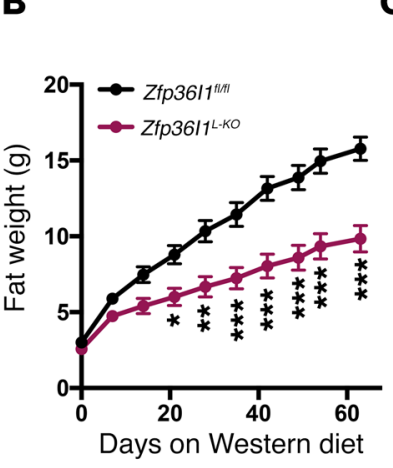

D

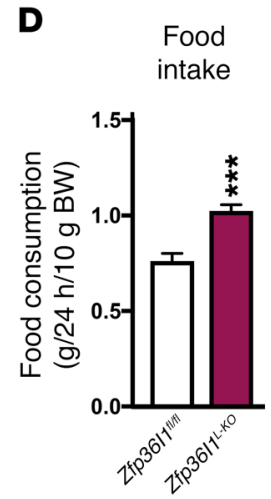

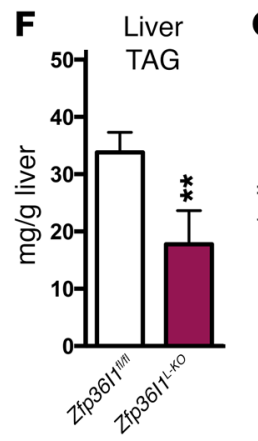

J

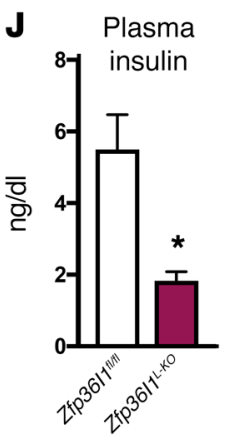

G Liver $\mathbf{H}$ Plasma I

4- cholesterol

K Plasma L

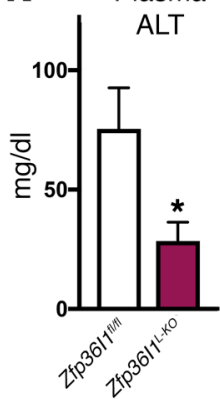

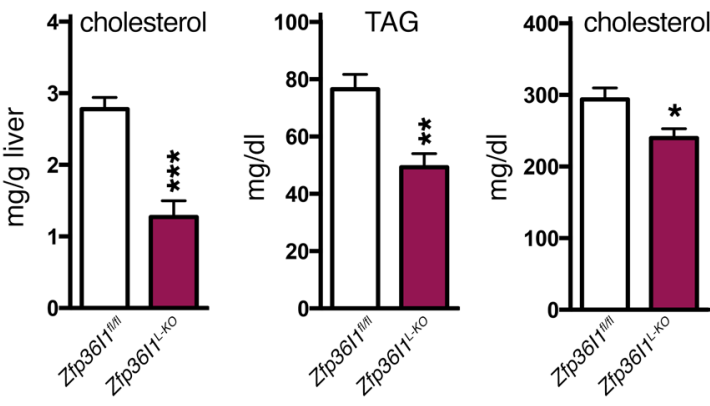

Plasma ${ }^{400}-$ cholesterol

M
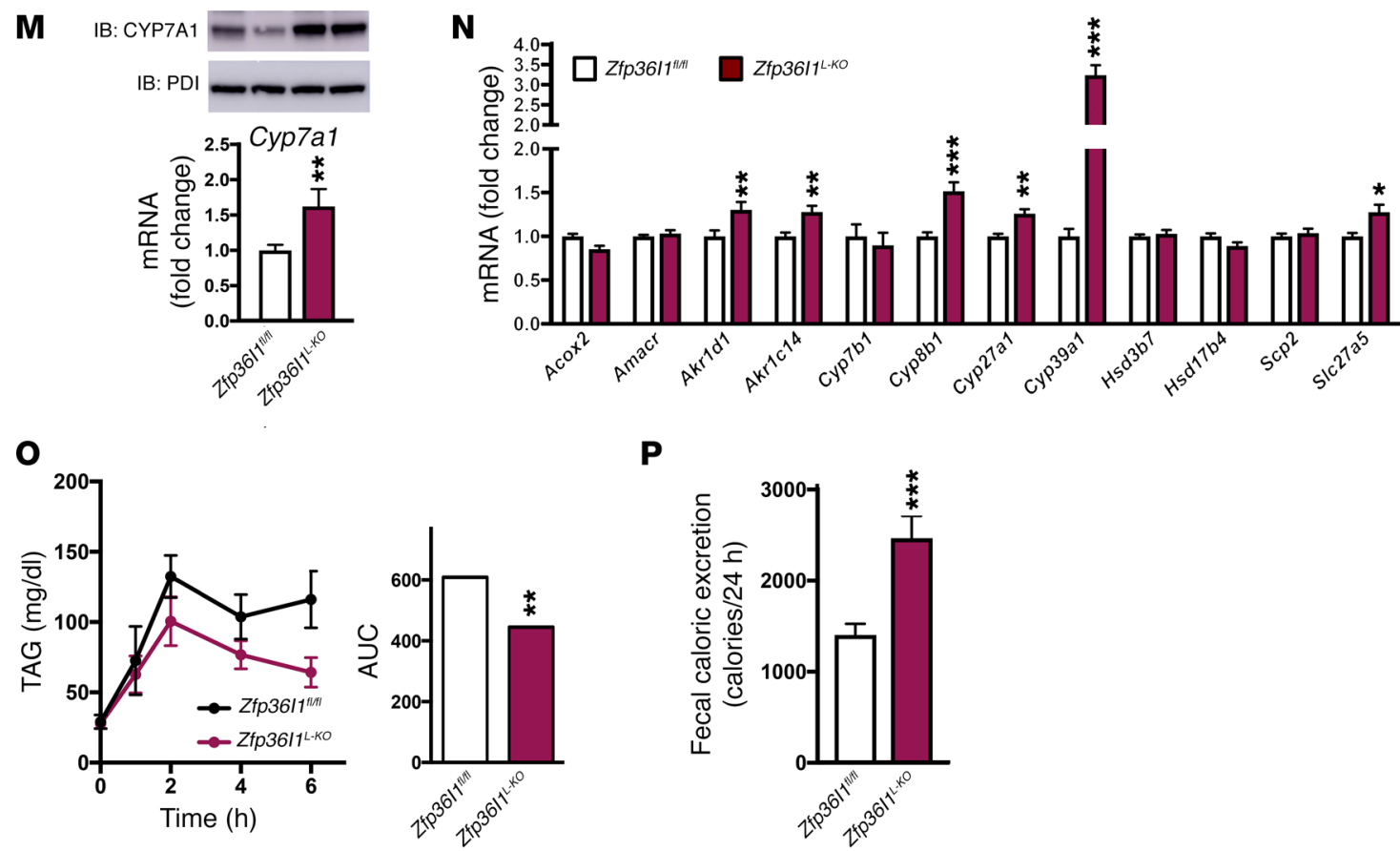

$\mathbf{P}$

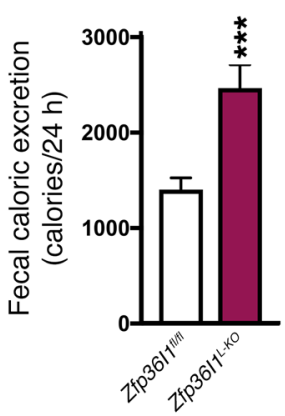


Figure 8. Loss of hepatic Zfp36/1 results in resistance to diet-induced obesity, protection from steatosis, and reduced lipid absorption. (A) Body weight $(\mathrm{g})$ and $(\mathbf{B})$ fat mass $(\mathrm{g})$ of male littermate $\mathrm{Zfp} 36 / /^{f / / f l}(n=31)$ and Zfp36/1 $1^{-K O}(n=19)$ mice measured weekly over a 64-day period of Western diet feeding. (C) Energy expenditure of male littermate $Z f p 36 / 7^{f / f l}$ $(n=5)$ and $Z f p 36 / 7^{-K O}(n=6)$ mice fed a Western diet for 64 days. (E) Representative images of livers and $\mathrm{H} \& \mathrm{E}$-stained sections from littermate pairs of $Z f p 36 / 7^{f / f l}$ and $Z f p 36 / 1^{-k O}$ mice fed a Western diet. Levels of (F) Hepatic triglyceride (TAG), (C) hepatic cholesterol, (H) plasma TAG, (I) plasma cholesterol, (J) plasma insulin, and (K) plasma ALT in littermate pairs of Zfp36/7 fl/fl and Zfp36/1-Ko mice fed a Western diet ( $n=19-10$ mice/ genotype). (L) Biliary bile acid levels and bile acid species, (M) CYP7A1 mRNA and protein levels, and (N) mRNA levels of other bile acid synthesis genes in littermate $Z f p 36 / 7^{f / f l}$ and $Z f p 36 / 7^{-K O}$ mice fed a Western diet $(n=$ 19-10 mice/genotype). (0) Plasma TAC levels following an intragastric fat challenge of male littermate $Z f p 36 / 7^{f / / f l}(n=6)$ and $Z f p 36 / 1^{-K o}$ mice $(n=7)$ fed a Western diet. Graph at right shows the AUC. (P) Fecal calorimetric data expressed as calories per 24-hour period for feces from $Z f p 36 / 1^{f / / f l}$ $(n=12)$ and $Z f p 36 / 7^{-K O}$ mice $(n=9)$. Data represent the mean \pm SEM. ${ }^{*} P<0.05$, ${ }^{* *} P<0.01$, and ${ }^{* *} P<0.001$, by 2 -way, repeated-measures ANOVA ( $\mathbf{A}$ and $\left.\mathbf{B}\right)$, repeated-measures 2-way ANCOVA (C), and Student's $t$ test (D-P).

Our gain- and loss-of-function studies show that there are significant metabolic consequences to modulation of Zfp36l1. Hepatic overexpression of $Z f p 36 l 1$ in mice resulted in a decrease in both Cyp7a1 and Cyp8b1 mRNA levels. Complementary lossof-function studies demonstrated that loss of Zfp36l1 in the livers of mice results in an elevated expression of a number of mRNAs, including Cyp7a1 and Cyp8b1, in both normal and Western dietfed mice. Consistent with these findings, Zfp $36 l 1^{L-K O}$ mice have increased biliary bile acid levels and an altered biliary bile acid composition. These results are consistent with the hypothesis that Zfp36l1 normally functions to repress Cyp7a1 mRNA and reduce bile acid synthesis. The reduction in plasma cholesterol levels that accompanied the increased bile acid levels is also consistent with increased bile acid synthesis in $Z f p 36 l 1^{L-K O}$ mice.

One of the major functions of bile acids is to facilitate lipid absorption, and it is clear that not all bile acids act in the same manner $(1,2)$. For example, Cyp $8 b 1^{-/-}$mice have increased T- $\beta$-MCA levels and no detectable tauro-CA. These same $C y p 8 b 1^{-/-}$mice have increased CYP7A1 enzymatic activity and an increased bile acid pool, yet decreased cholesterol absorption (7). Interestingly, Cyp $8 b 1^{-/-}$mice were recently shown to be resistant to steatosis and weight gain (32). The improved steatosis and decreased adiposity are similar to our findings in $Z f p 36 l 1^{L-K O}$ mice, which also had increased bile acid levels and, in particular, increased levels of T- $\beta$ MCA. Thus, the different bile acid composition of $Z f p 36 l 1^{L-K O}$ mice may account, at least in part, for the decrease in lipid absorption and the increase in fecal calorie content as well as the resistance to diet-induced adiposity and the decrease in steatosis. While the changes in plasma triglycerides following an oral lipid challenge were modest, the changes in body weight and adiposity are likely reflective of cumulatively defective lipid absorption over the 64-day period that the animals were on the diet.

Previous studies have defined two different FXR-dependent pathways, one requiring hepatic SHP and the other being dependent on intestinally derived FGF15/19, that are involved in the repression of Cyp7a1 transcription $(8,9)$. Both these pathways have also been shown to work together, as recombinant FGF19 was unable to reduce Cyp7a1 mRNA levels in $S h p^{-/-}$mice (11). We recently described an FXR-dependent pathway that functions through another transcriptional repressor, MAFG, which does not directly repress $C y p 7 a 1$. MAFG functions to repress numerous genes in the bile acid synthesis pathway, including Cyp8b1, the regulatory gene of CA synthesis (14). The relative contribution of each of these pathways to feedback regulation of bile acid synthesis has not been comprehensively established. Previous studies using $S h p^{-/-}$mice showed that treatment with GW4064 failed to repress Cyp7a1 mRNA levels $(33,34)$, supporting the idea that SHP has a role in FXR-dependent repression of this gene. In contrast, we and others have reported that Cyp7a1 mRNA levels decline following treatment of Shp ${ }^{-/-}$mice with either GW4064 (35), CA $(33,34)$, or GSK2324, as we have shown here. At present, it is unclear whether these divergent results are due to the different genetic backgrounds of the animal models or to differences in the modes of administration, tissue uptake, or pharmacokinetics of the FXR agonists. Consequently, the exact role and importance of SHP in the repression of Cyp $7 a 1$ remains to be clearly elucidated, although our data suggest that SHP is not essential for feedback regulation of bile acid synthesis.

The finding that treatment of $Z f p 36 l 1{ }^{L-K O}$ mice with GSK2324 results in reduced $C y p 7 a 1$ expression, albeit at a slower rate than in their WT littermates, suggests that Zfp36l1 is also not essential for feedback regulation of bile acid synthesis. Nonetheless, the findings that hepatic loss of ZFP36L1 results in elevated levels of Cyp7a1 and bile acids, reduced adiposity, and steatosis indicate that this protein functions in controlling Cyp7a1 mRNA. Importantly, here we show that GSK2324 treatment of WT, $S h p^{-/-}$, and Zfp36l1 ${ }^{L-K O}$ mice reduces Cyp7a1 mRNA levels. In contrast, Cyp7a1 mRNA levels were unaltered in $\mathrm{Fxr}^{-1-}$ mice treated with GSK2324. Taken together, we conclude that FXR utilizes multiple redundant pathways to mediate feedback inhibition of bile acid synthesis. Redundancy in feedback inhibition of bile acid synthesis was also proposed in the original reports characterizing $\operatorname{Sh} p^{-/-}$mice $(33,34)$.

Our identification of ZFP36L1 as a regulator of Cyp7a1 mRNA and bile acid composition provides a pathway that complements the known transcriptional repression pathways that decrease bile acid synthesis. ZFP36L1 may function to rapidly decrease preexisting Cyp7a1 mRNA levels, and subsequent transcriptional repression mechanisms in response to FGF15 and/or SHP then maintain reduced levels of Cyp7a1 mRNA under conditions of elevated bile acids and/or FXR activation. In addition to ZFP36L1, we have identified at least 1 other RBP that appears to be FXR regulated and can target and degrade Cyp7a1 mRNA (data not shown). However, we have not yet characterized this pathway in detail. It remains to be determined whether the different RBPs that are responsive to FXR activation target $C y p 7 a 1$ mRNA function in concert.

Studies conducted over a decade ago by Davis and colleagues had proposed that $C y p 7 a 1$ might be regulated by posttranscriptional mechanisms (24). However, no molecular mechanism was identified at that time, although the AU-rich elements present in the Cyp7a1 UTR were found to be required for the proposed posttranscriptional regulation (24). Since ZFP36L1 requires these AU-rich elements to repress Cyp7a1 mRNA levels, we believe we have identified a factor involved in the posttranscriptional regulation of Cyp7a1. 
ZFP36L1 can bind RNA directly but does not have the capability of degrading mRNAs by itself. Rather, ZFP36L1 binds mRNA targets in a sequence-specific fashion and then recruits additional proteins, including deadenylases, that then mediate mRNA degradation (13). Thus, ZFP36L1 acts as a guide for deadenylase complexes by recognizing specific motifs on mRNAs that are to be targeted for degradation. ZFP36L1 is known to bind AU-rich elements, which are common in 3'-UTRs of many genes, including Cyp7a1. However, it is clear that not all genes that contain AU-rich elements are regulated in the same way. The mRNA targets of ZFP36L1 are probably cell specific and depend not only on the presence of other specific proteins but also on the abundance of target mRNAs. Thus, the specificity of ZFP36L1 for particular targets is likely a combination of mRNA sequences and secondary structure, mRNA abundance, and protein-protein interactions. Additional studies will be required to identify and characterize the protein components in the liver that are involved in regulating Cyp7a1 mRNA stability and degradation.

ZFP36L1 has also been implicated in human disease, as GWAS identified variants in the ZFP36L1 locus associated with Crohn's disease and inflammatory bowel disease (IBD) (36). Since altered bile acid metabolism has also been implicated in diseases of the gut, our studies may provide an additional mechanism to explain, at least in part, how variants in the ZFP36L1 locus are associated with IBD and Crohn's disease.

Bile acids are both signaling molecules and detergents, thus, we also determined whether loss of $Z f p 36 l 1$ in the liver results in broader metabolic dysfunction. Compared with their WT littermates, Zfp $36 l 1^{L-K O}$ mice had markedly reduced obesity, without changes in energy expenditure compared with their littermate WT controls. Zfp36l1 ${ }^{L-K O}$ mice were also protected from diet-induced hepatosteatosis. We propose that these metabolically beneficial effects are due to defects in lipid absorption, since $Z f p 36 l 1^{L-K O}$ mice had a reduced response following an intragastric fat challenge and increased fecal calorie output. Interestingly, CYP7A1-transgenic mice exhibit many of the same phenotypes we have described here in $Z f p 36 l 1^{L-K O}$ mice, including resistance to obesity and protection from steatosis (28-30). However, the mechanisms leading to the changes in obesity, steatosis, and lipid absorption in CYP7A1-transgenic mice remain unknown (28-30). Nonetheless, our studies suggest that many of the phenotypic changes we observed in Zfp36l1 ${ }^{\mathrm{L} K O}$ mice were a direct result of increased CYP7A1 activity, increased bile acid synthesis, and an altered bile acid pool size and composition that then affected lipid absorption in the gut. The prevalence of nonalcoholic fatty liver disease (NAFLD) and obesity has increased worldwide, and the pathogenesis of NAFLD is poorly understood (37). Our studies suggest that dysregulated bile acid metabolism is probably important in NAFLD progression.

\section{Methods}

Animals and treatments. All animals were bred and housed in a pathogen-free animal facility. C57BL/6 liver- and intestine-specific $\mathrm{Fxr}^{-/-}$ mice and their respective (littermate) Fx $\mathrm{r}^{\mathrm{fl} / \mathrm{fl}} \mathrm{WT}$ controls as well as whole-body $\mathrm{Fxr}^{-1-}$ mice were generated as previously described (20). C57BL/6 littermate $Z f p 36 l 1^{f l-f l}(27)$ and $Z f p 36 l 1^{L-K O}$ mice were generated by crossing $Z f p 36 l 1^{f / f l}$ mice (31) with albumin-Cre-expressing animals (The Jackson Laboratory). Whole-body $S h p^{-/-}$mice (17) were obtained and backcrossed at UCLA for 6 further generations on a C57BL/ 6 back- ground. All mice were maintained on a 12-hour light/12-hour dark cycle. In general, animals were used at 60 days of age, fasted at 9 am for 4 to 5 hours, and then sacrificed between $1 \mathrm{pm}$ and $2 \mathrm{pm}$. For treatments with FXR agonists, GSK2324 was dissolved in water and administered once to mice via i.p. injection at $30 \mathrm{mpk}$ for 30 minutes, 1 hour, 2 hours, or 4 hours before sacrifice. Vehicle-treated mice were injected with water for randomly assigned time points (between $30 \mathrm{~min}$ and $4 \mathrm{~h}$ ). All mice were fasted for 4 hours and sacrificed at the same time of day (1-3 pm) to ensure a consistent circadian time point. Studies with GW4064 and GSK2324 at 60 mpk for 3 days were described previously (14).

Adenovirus production. All adenoviruses were prepared in biosafety level 2-category (BSL-2-category) facilities. Briefly, cDNAs for mouse $Z f p 36 l 1$ were cloned from whole-liver cDNA into a pAdTrack CMV plasmid and prepared as described previously (14). For animal experiments, $1 \times 10^{9} \mathrm{PFU}$ were infused into either male or female C57BL/ 6 mice for 5 to 7 days before tissue collection.

Bile acid analysis. Gall bladders were removed after a 4- to 6-hour fast, bile was removed, and the contents were stored at $-80^{\circ} \mathrm{C}$. Bile acid species were measured using an HPLC system as described previously (14). Conjugated bile acids were analyzed by HPLC (Shimadzu) using a Kinetex 5m C-18 100A $250 \times 4.6 \mathrm{~mm}$ column (Phenomenex) with isocratic elution at $0.75 \mathrm{ml} / \mathrm{min}$. The eluting solution was composed of a mixture of methanol and $0.01 \mathrm{M} \mathrm{KH}_{2} \mathrm{PO}_{4}(67.4 \% \mathrm{v} / \mathrm{v})$, adjusted to an apparent $\mathrm{pH}$ of 5.25 with $\mathrm{H}_{2} \mathrm{PO}_{4}$. Bile acids were quantified by measuring their absorbance at $205 \mathrm{~nm}$ and identified by matching their relative retention times with those of known standards.

Total bile acids were quantified with a calorimetric assay (Diazyme) using standards prepared with Na-tauro-CA. For biliary bile acids, samples were diluted at 1:1,000, and $10 \mu \mathrm{l}$ was assayed. Bile acid amounts were calculated by multiplying the concentrations per microliter with the volume of bile in each gall bladder.

RNA isolation and quantitative real-time PCR. Liver samples (approximately $100 \mathrm{mg}$ ) were removed from mice and immediately flash frozen in liquid nitrogen and then stored at $-80^{\circ} \mathrm{C}$. Frozen tissue was homogenized in QIAzol (QIAGEN) and extracted according to the manufacturer's instructions. RNA was then treated with DNAse (rDNAseI; Ambion, Life Technologies, Thermo Fisher Scientific) for 1 hour at $37^{\circ} \mathrm{C}$ and reextracted by phenol/chloroform/isoamyl alcohol (25:24:1, pH 6.6; Life Technologies, Thermo Fisher Scientific). cDNA was synthesized from 500 ng RNA using the High Capacity cDNA Reverse Transcriptase Kit (Applied Biosciences, Thermo Fisher Scientific) according to the manufacturer's instructions. Quantitative real-time PCR (qRT-PCR) standards were prepared from an aliquot from each $\mathrm{cDNA}$ reaction, which was then pooled. Standards were then diluted over a 3-log range (dilution range of 1:5, 1:10, 1:50, 1:100, 1:500, and 1:1,000). qRT-PCR was performed with primers designed to cross exon-exon boundaries using the Roche UPL primer design website (https://lifescience.roche.com/en_us/brands/ universal-probe-library.html). qPCR was performed in triplicate for each sample in a 384-well format using Kapa LC480 SYBR Green Mix (Kapa Biosystems). qPCR was performed using a Lightcycler 480 (Roche), and concentrations were determined from the standard curve using the efficiency-corrected method (second derivative maximum, Roche). Relative quantification was determined by normalizing the expression of each gene to a housekeeping gene. Two housekeeping genes (36B4 and $T b p$ ) were used to normalize gene expression data. The primer sequences were previously described (14), except for Zfp36l1 (forward: TTCACTACACACCAGATCCT; reverse: TGAGCAtCTTGTTACCCTTGC). 
FXR agonist treatments and mRNA half-life determination in cultured cells. IHHs were seeded in 6-well plates and treated with $5 \mathrm{mg} /$ $\mathrm{ml}$ actinomycin D (Sigma-Aldrich) dissolved in DMSO for various time intervals. All cells treated with actinomycin D for various time periods were harvested at the same time. CDCA (Sigma-Aldrich) or GW4064 (Sigma-Aldrich) was dissolved in vehicle (DMSO) and added to cells plated at $70 \%$ to $80 \%$ confluency. IHHs were treated at the concentrations and for the durations specified in the figure legends. Cells were then washed in PBS and harvested in QIAzol, and RNA was isolated using a miRNeasy Mini Kit (QIAGEN) according to the manufacturer's instructions. Gene expression was determined as described above. The primer sequences were previously described (14), except for huZFP36L1 (forward: GATGACCACCACCCTCGT; reverse: TGGGAGCACTATAGTTGAGCATC).

Western blot analysis. Liver samples (approximately $100 \mathrm{mg}$ ) were homogenized in $1 \mathrm{ml}$ RIPA buffer supplemented with protease inhibitor complex (Cell Signaling Technology). Protein was quantified using the BCA assay (Thermo Fisher Scientific), and $50 \mu \mathrm{g}$ protein was used for Western blot analysis. PVDF membranes (EMD Millipore) were probed with antibodies overnight, and HRP detection was performed using ECL Reagent (Sigma-Aldrich) or ECL Prime (GE Healthcare) according to the manufacturers' instructions. Anti-ZFP36L1 antibody (BRF1/2, catalog 2119; Cell Signaling Technology; 1:1,000); anti-CYP7A1 antibody (catalog MABD42; Sigma-Aldrich; 1:1,000); protein disulfide isomerase (PDI) (catalog 3501; Cell Signaling Technology; 1:1,000); and secondary anti-rabbit HRP-conjugated antibody (GE Healthcare; 1:10,000) were used. HRP signal detection was determined electronically using an Amersham Imager 600UV system (GE Healthcare), and exposure was determined using GE software strictly set below the saturation point.

Promoter and UTR reporter analysis. A mouse Zfp36l1 promoter $(2 \mathrm{~kb})$ luciferase reporter construct was generated by amplifying these regions from mouse genomic DNA using KAPA HiFi polymerase (Kapa Biosystems) and cloning them into a pGL4.10[luc2] plasmid (Promega). Mouse or human Cyp7a1 UTR sequences were cloned into pcDNA3.1(+) containing luciferase (luc2 gene; Promega) inserted at the beginning of the multiple cloning site. Luciferase reporter constructs were transfected using Fugene HD (Promega), according to the manufacturer's instructions, into human IHHs plated onto 48 -well dishes ( $n=6$ wells/condition). After 24 hours, medium was replaced, and for promoter reporter experiments, cells were treated with either vehicle (DMSO) or GW4064 (1 $\mu \mathrm{M}$ in DMSO) in medium containing $10 \%$ charcoal-stripped serum (Omega Scientific) for a further 24 hours.

Diet feeding studies and metabolic analysis. The Western-style diets (high-fat, high-cholesterol; catalog D12079B) and high-fat diets (cata$\log$ D12451) were purchased from Research Diets. CA-containing diets (0.5\%) and global control diets were purchased from Envigo (Custom Diets). Body composition was determined weekly using NMR (Bruker Minispec) by measuring body fat mass and lean mass. For metabolic analysis, oxygen consumption, carbon dioxide production, and respiratory exchange ratio were determined using a modified open-circuit calorimeter (comprehensive laboratory animal monitoring system [CLAMS]; Columbus Instruments). Data for $\mathrm{VO}_{2}, \mathrm{VCO}_{2}$, and RER were used to calculate energy expenditure, as described previously (38).

Food consumption and calorimetric analysis. Littermate Zfp36lit/fl and $Z f p 36 l 1^{L-K O}$ mice were fed a Western diet for 64 days and then individually housed for 2 days in cages containing metal grills to allow for acclimation. After 48 hours, mice were moved to a new clean cage with a metal grill, and food consumption was monitored every 24 hours for 48 hours. After 48 hours, fecal content was collected, dried, and weighed. Bomb calorimetric analysis of dried fecal samples was performed at the UT Southwestern Metabolic Phenotyping Core. Calories are expressed per gram of feces and per total mass excreted for each animal.

Plasma and liver lipid analysis. Plasma lipids were analyzed as previously described (20). For hepatic lipid analysis, liver tissue was snap frozen in liquid nitrogen, and total lipids were extracted by a modified Folch extraction method (39). Briefly, 50-100 mg liver tissue was homogenized in methanol. Lipids were extracted overnight in chloroform/methanol (2:1, v/v) and filtered. The extraction tube was rinsed twice with chloroform/methanol $(2: 1, \mathrm{v} / \mathrm{v})$ and combined with the filtrate. To induce phase separation, $0.043 \%$ magnesium chloride was added, and the upper aqueous phase and interface (containing salted out proteins) were removed. The lower organic phase was dried under a nitrogen stream. The dried lipid was resuspended in $1 \%$ Triton $\mathrm{X}-100$ in chloroform ( $3 \mathrm{ml}$ per $0.1 \mathrm{~g}$ tissue) and dried down once more under a nitrogen stream. The dried lipid was resuspended in $1.8 \%$ Triton X-100 in water for lipid quantification using a calorimetric triglyceride or cholesterol assay kit (Thermo Fisher Scientific). Liver sections were prepared from paraformaldehyde tissue fixed overnight at the UCLA Translational Pathology Core Laboratory (TPCL) and stained with $\mathrm{H} \& \mathrm{E}$.

Plasma metabolites and enzymes. EDTA plasma samples from animals were stored at $-80^{\circ} \mathrm{C}$ after collection. Plasma ALT and AST (Teco Diagnostics) and plasma glucose levels were determined according to the manufacturer's instructions (Stanbio Laboratory). Plasma insulin levels were determined by ELISA (Alpco).

Statistics. All error bars represent the mean \pm SEM. Significance was calculated using a 2-tailed, paired Student's $t$ test for comparison of 2 groups (Microsoft Excel) or a 1- or 2-way ANOVA (GraphPad Prism, GraphPad Software) for comparison of more than 2 groups. For dietary studies over time, we used a 2-way repeated-measures ANOVA (GraphPad Prism Software). A repeated-measures 2-way ANCOVA (SPSS Inc.) was performed for analysis of metabolic parameters, with body weight, fat mass, and lean mass as defined covariates. Where Mauchly's test for sphericity failed, significance was calculated using the Greenhouse-Geisser correction. A P value of less than 0.05 was considered statistically significant.

Study approval. All animal experiments were performed according to NIH guidelines and were approved by the Office of Animal Research Oversight (OARO) at UCLA.

\section{Author contributions}

Study concept and design: TDAV; data acquisition: EJT, BLC, JC, AC, EL, PM, and TDAV; data analysis and interpretation: EJT, BLC, and TDAV; statistical analysis: BLC and TDAV; CLAMS: BLC and TS; mouse model: MT; technical support: JC, AC, EL, and PM; writing of the manuscript: EJT and TDAV; revision of the manuscript: EJT, TS, MT, and TDAV; study supervision: TDAV.

\section{Acknowledgments}

We thank Peter Edwards for his guidance and input on this manuscript. We also thank Peter Tontonoz and Steve Bensinger and the members of their laboratories (UCLA) for feedback and suggestions. We thank Tim Willson and David Deaton (GSK, 
Research Triangle Park, North Carolina, USA) for GSK2324; Bryan Goodwin and Helen Hartman (Pfizer, Collegeville, Pennsylvania, USA) for the $S h p^{-/-}$mice; Richard Davis (UCLA) for help with gavage experiments; and Laurent Vergnes (UCLA) for the IHHs. EJT is supported by NIH grants HL118161 and HL136543; TS is supported by NIH grant HL128822; MT is supported by Biotechnology and Biological Sciences Research Council grants BB/ J004472/1 and BB/J00152X/1; and TQDAV is supported by NIH grants HL122677 and DK112119 and in part by HL028481 and
DK102559. TQDAV was also supported by the UCLA Clinical and Translational Science Institute (UL1TR000124); the UCLA/ UCSD Diabetes Research Center (DK063491); and the American Heart Association (SDG18440015).

Address correspondence to: Thomas Q. de Aguiar Vallim, UCLA Division of Cardiology, 650 Charles E. Young Drive South, A2-237 CHS (MC167917), Los Angeles, California 90095, USA. Email: tvallim@mednet.ucla.edu.
1. de Aguiar Vallim TQ, Tarling EJ, Edwards PA. Pleiotropic roles of bile acids in metabolism. Cell Metab. 2013;17(5):657-669.

2. Russell DW. The enzymes, regulation, and genetics of bile acid synthesis. Annu Rev Biochem. 2003;72:137-174.

3. Makishima $\mathrm{M}$, et al. Identification of a nuclear receptor for bile acids. Science. 1999;284(5418):1362-1365.

4. Parks DJ, et al. Bile acids: natural ligands for an orphan nuclear receptor. Science. 1999;284(5418):1365-1368.

5. Sayin SI, et al. Gut microbiota regulates bile acid metabolism by reducing the levels of tauro-betamuricholic acid, a naturally occurring FXR antagonist. Cell Metab. 2013;17(2):225-235.

6. Neuschwander-Tetri BA, et al. Farnesoid X nuclear receptor ligand obeticholic acid for noncirrhotic, non-alcoholic steatohepatitis (FLINT): a multicentre, randomised, placebo-controlled trial. Lancet. 2015;385(9972):956-965.

7. Li-Hawkins J, et al. Cholic acid mediates negative feedback regulation of bile acid synthesis in mice. J Clin Invest. 2002;110(8):1191-1200.

8. Goodwin B, et al. A regulatory cascade of the nuclear receptors FXR, SHP-1, and LRH-1 represses bile acid biosynthesis. Mol Cell. 2000;6(3):517-526.

9. Inagaki $\mathrm{T}$, et al. Fibroblast growth factor 15 functions as an enterohepatic signal to regulate bile acid homeostasis. Cell Metab. 2005;2(4):217-225.

10. Kliewer SA, Mangelsdorf DJ. Bile Acids as Hormones: The FXR-FGF15/19 Pathway. Dig Dis. 2015;33(3):327-331.

11. Kir S, Zhang Y, Gerard RD, Kliewer SA, Mangelsdorf DJ. Nuclear receptors HNF $4 \alpha$ and LRH-1 cooperate in regulating Cyp7a1 in vivo. J Biol Chem. 2012;287(49):41334-41341.

12. Varnum BC, Ma QF, Chi TH, Fletcher B, Herschman HR. The TIS11 primary response gene is a member of a gene family that encodes proteins with a highly conserved sequence containing an unusual Cys-His repeat. Mol Cell Biol. 1991;11(3):1754-1758.

13. Newman R, McHugh J, Turner M. RNA binding proteins as regulators of immune cell biology. Clin Exp Immunol. 2016;183(1):37-49.

14. de Aguiar Vallim TQ, et al. MAFG is a transcriptional repressor of bile acid synthesis and metabolism. Cell Metab. 2015;21(2):298-310.
15. Andreou ER, Prokipcak RD. Analysis of human CYP7A1 mRNA decay in HepG2 cells by reverse transcription-polymerase chain reaction. Arch Biochem Biophys. 1998;357(1):137-146.

16. Schippers IJ, et al. Immortalized human hepatocytes as a tool for the study of hepatocytic (de-)differentiation. Cell Biol Toxicol. 1997;13(4-5):375-386.

17. Hartman HB, Lai K, Evans MJ. Loss of small heterodimer partner expression in the liver protects against dyslipidemia. J Lipid Res. 2009;50(2):193-203.

18. Gerstberger S, Hafner M, Tuschl T. A census of human RNA-binding proteins. Nat Rev Genet. 2014;15(12):829-845.

19. Bartel DP. MicroRNAs: target recognition and regulatory functions. Cell. 2009;136(2):215-233.

20. de Aguiar Vallim TQ, et al. MicroRNA-144 regulates hepatic ATP binding cassette transporter A1 and plasma high-density lipoprotein after activation of the nuclear receptor farnesoid $\mathrm{X}$ receptor. Circ Res. 2013;112(12):1602-1612.

21. Tarling EJ, Ahn H, de Aguiar Vallim TQ. The nuclear receptor FXR uncouples the actions of miR-33 from SREBP-2. Arterioscler Thromb Vasc Biol. 2015;35(4):787-795.

22. Thomas AM, Hart SN, Kong B, Fang J, Zhong XB, Guo GL. Genome-wide tissue-specific farnesoid $\mathrm{X}$ receptor binding in mouse liver and intestine. Hepatology. 2010;51(4):1410-1419.

23. Chong HK, et al. Genome-wide interrogation of hepatic FXR reveals an asymmetric IR-1 motif and synergy with LRH-1. Nucleic Acids Res. 2010;38(18):6007-6017.

24. Baker DM, Wang SL, Bell DJ, Drevon CA, Davis RA. One or more labile proteins regulate the stability of chimeric mRNAs containing the 3'-untranslated region of cholesterol7alpha -hydroxylase mRNA. J Biol Chem. 2000;275(26):19985-19991.

25. Stumpo DJ, et al. Chorioallantoic fusion defects and embryonic lethality resulting from disruption of Zfp36L1, a gene encoding a CCCH tandem zinc finger protein of the Tristetraprolin family. Mol Cell Biol. 2004;24(14):6445-6455.

26. Bell SE, et al. The RNA binding protein Zfp 3611 is required for normal vascularisation and post-transcriptionally regulates VEGF expression. Dev Dyn. 2006;235(11):3144-3155.

27. Hodson DJ, et al. Deletion of the RNA-binding proteins ZFP36L1 and ZFP36L2 leads to perturbed thymic development and T lymphoblastic leukemia. Nat Immunol. 2010;11(8):717-724.

28. Miyake JH, et al. Increased production of apolipoprotein B-containing lipoproteins in the absence of hyperlipidemia in transgenic mice expressing cholesterol 7alpha-hydroxylase. J Biol Chem. 2001;276(26):23304-23311.

29. Li T, et al. Overexpression of cholesterol $7 \alpha$-hydroxylase promotes hepatic bile acid synthesis and secretion and maintains cholesterol homeostasis. Hepatology. 2011;53(3):996-1006.

30. Li T, Owsley E, Matozel M, Hsu P, Novak CM, Chiang JY. Transgenic expression of cholesterol 7alpha-hydroxylase in the liver prevents high-fat diet-induced obesity and insulin resistance in mice. Hepatology. 2010;52(2):678-690.

31. Galloway A, et al. RNA-binding proteins ZFP36L1 and ZFP36L2 promote cell quiescence. Science. 2016;352(6284):453-459.

32. Bonde Y, Eggertsen G, Rudling M. Mice Abundant in Muricholic Bile Acids Show Resistance to Dietary Induced Steatosis, Weight Gain, and to Impaired Glucose Metabolism. PLOS ONE. 2016;11(1):e0147772.

33. Wang L, et al. Redundant pathways for negative feedback regulation of bile acid production. Dev Cell. 2002;2(6):721-731.

34. Kerr TA, et al. Loss of nuclear receptor SHP impairs but does not eliminate negative feedback regulation of bile acid synthesis. Dev Cell. 2002;2(6):713-720.

35. Kong B, Wang L, Chiang JY, Zhang Y, Klaassen CD, Guo GL. Mechanism of tissue-specific farnesoid $\mathrm{X}$ receptor in suppressing the expression of genes in bile-acid synthesis in mice. Hepatology. 2012;56(3):1034-1043.

36. Liu JZ, et al. Association analyses identify 38 susceptibility loci for inflammatory bowel disease and highlight shared genetic risk across populations. Nat Genet. 2015;47(9):979-986.

37. Cohen JC, Horton JD, Hobbs HH. Human fatty liver disease: old questions and new insights. Science. 2011;332(6037):1519-1523.

38. Tschöp MH, et al. A guide to analysis of mouse energy metabolism. Nat Methods. 2011;9(1):57-63.

39. Folch J, Lees M, Sloane Stanley GH. A simple method for the isolation and purification of total lipides from animal tissues. J Biol Chem. 1957;226(1):497-509. 